\title{
On the controllability of the Laplace equation observed on an interior curve.
}

\author{
A. OSSES ${ }^{1}$ and J.- P. PUFI
}

\begin{abstract}
The boundary approximate controliability of the Laplace equation observed on an interior curve is studied in this paper. First we consider the Laplace equation with a bounded potential. The $L^{p}$ $(1<p<\infty)$ approximate controllability is established and controls of $L^{p}$-minimal norm are built by duality. At this point, a general result which clarifies the relationship between this duality approach and the classical optimal control theory is given. The results are extended to the $L^{p} \quad( \pm \leq p<\infty)$ approximate controllability with quasi bang-bang controls and finally to the semilinear case with a globally Lipschitz non linearity by a fixed point method. A counterexample shows that the globally Lipschitz hypothesis is essential. To compute the control, a numerical method based in the duality technique is proposed. It is tested in several cases obtaining a fast behaviour in the case of fixed geometry.
\end{abstract}

\section{Introduction and Main Results}

\subsection{Introduction}

In this paper we present some extensions and numerical applications of a method which has been developed by Fabre, Puel and Zuazua in

${ }^{1}$ The author gratefully acknowledges the support of the French Scientific Committee ECOS.

AMS Classifications: 35B37, 93B40, 93B06, 93C10.

Servicio Publicaciones Univ. Complutense, Madrid, 1998. 
a series of papers [Fa-Pu-Zu 1,2,3]. They studied the boundary and internal approximate controllability of the semilinear heat equation observed at final time. We consider here the boundary control of the semilinear Laplace equation observed on an internal surface (also see [Os-Pu1]). The boundary value is unknown on a part of the boundary but some "measurements" of the solution in $L^{p}$ are given on the internal surface. The problem is then to retrieve the boundary value from the given measurements. This can be viewed as an inverse problem to which approximate controllability techniques apply.

By definition, if we can find an approximation of the boundary value we say that we have $L^{p}$-approximate controllability. In this paper, we study in an unified manner the $L^{p}$-approximate controllability for $1<$ $p<\infty$ with controls of minimal $p$-norm, the case of quasi bang-bang controls, the semilinear case and a numerical resolution of the problem.

Our results make use of unique continuation properties which are classical in the case of the Laplace equation. 'Thanks to recent unique continuation properties of the generalized Stokes system [Fa-Le], we treat in another paper the Stokes case for which numerical calculations are also given [Os-Pu2].

\subsection{Control Problem}

Let $\Omega$ be a regular bounded open set of $\mathbb{R}^{N}, N \geq 2$. We also suppose that $\Omega$ is connected, in fact the results of this paper are valid in each connected component of $\Omega$ separately.

We denote by $\Gamma$ the boundary of $\Omega$, and by $\nu$ the unit outward normal of $\Omega$. We suppose that $\Gamma_{0}$ is a non-empty and relatively open part of $\Gamma$ which represents the boundary where the control acts. We are now given an internal regular subset $S$ of $\Omega$ of codimension 1, that is to say a curve in two dimensions or a surface in three, where we observe or measure the solution. 


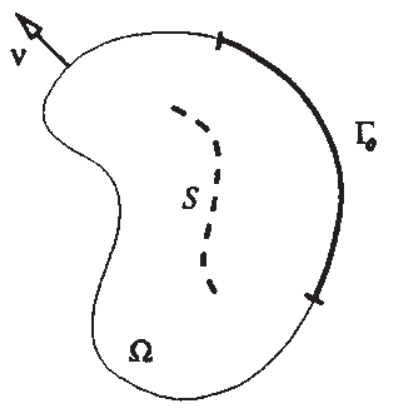

FIG. 1. Principal notations.

Let us consider for each $v \in L^{p}\left(\Gamma_{0}\right), 1<p<\infty$ the following Dirichlet boundary problem:

$$
\begin{array}{rll}
-\Delta y+a y=f & \text { in } & \Omega \\
y=v & \text { on } & \Gamma_{0} \\
y=0 & \text { on } & \Gamma \backslash \Gamma_{0},
\end{array}
$$

where $f \in L^{p}(\Omega)$ and the potential function $a$ satisfies the following conditions

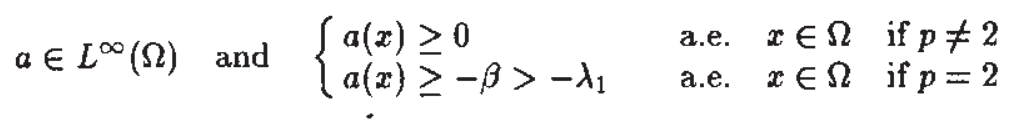

where $\lambda_{1}>0$ denotes the smallest eigenvalue of the $-\Delta$ operator in $\Omega$ with Dirichlet homogeneous boundary conditions. We will see that problem (1.1) has a unique solution $y=y(v)$ in a transposition sense [Li-Ma], but this solution is only in $L^{P}(\Omega)$. Nevertheless, the trace of $y(v)$ on $S$ still makes sense if $S$ is supposed to be strictly included in $\Omega$ as we will see later.

Given $y_{1} \in L^{p}(S)$ and $\alpha>0$, our aim is finding $v \in L^{p}\left(\Gamma_{0}\right)$ such that

$$
\left\|y(v)_{/ S}-y_{1}\right\|_{0, p, S} \leq \alpha
$$

where $\|\cdot\|_{0, p, S}$ denotes the standard norm in $L^{p}(S)$ (see section 1.4).

Of course, $\alpha=0$ would be the ideal case of exact controllability, but this is in general impossible due to regularity reasons. For example, if $f=0$ in $(1.1)$ then $y(v)$ is analytic in $\Omega$ and $y_{1}$ is only taken in $L^{p}(S)$. 
Condition (1.3) follows from classic approximate controllability methods since our problem is equivalent to showing that the set $\left\{y(v)_{/ S}, v \in\right.$ $\left.L^{p}\left(\Gamma_{0}\right)\right\}$ is dense in $L^{p}(S)$.

\subsection{Main results}

In order to obtain the approximate controllability results, we have to introduce the following geometrical hypothesis on $S$ and $\Gamma_{0}$ :

Sis strictly contained in $\Omega$ and each point on Scan be connected to $\Gamma_{0}$ by an are in $\Omega$ which does not cross $\bar{S}$.

For each $\varphi_{0} \in L^{p^{\prime}}(S)$, let us denote by $\varphi$ the solution of the problem

$$
\begin{aligned}
-\Delta \varphi+a \varphi & =\varphi_{0} \delta_{S} \quad \text { in } \quad \Omega \\
\varphi & =0 \text { on } \Gamma,
\end{aligned}
$$

where $\delta_{S}$ denotes the Dirac mass on $S$.

Finally, we define $\bar{y}$ as the solution of the following homogeneous problem:

$$
\begin{array}{rll}
-\Delta \bar{y}+a \bar{y}=f & \text { in } \quad \Omega \\
\bar{y}=0 & \text { on } \quad \Gamma .
\end{array}
$$

Theorem 1.1. Assume (1.4). For each $y_{1} \in L^{p}(S), 1<p<\infty$ and $\alpha>0$, there exists a control $v$ in $L^{p}\left(\Gamma_{0}\right)$ such that the solution $y(v)$ of the problem (1.1) satisfies (1.3). Moneover, if $\mathcal{U}_{a d}$ represents the set of all v in $L^{p}\left(\Gamma_{0}\right)$ which satisfies (1.3), called admissible controls, then the minimization problem:

$$
\min _{v \in \mathcal{U}_{a d}} \frac{1}{p}\|v\|_{0, p, S}^{p}
$$

has a unique solution $\hat{v}$. On the other hand, if we define the functional

$$
J\left(\varphi_{0}\right)=\frac{1}{p^{\prime}} \int_{\Gamma_{0}}\left|\frac{\partial \varphi}{\partial p^{\prime}}\right|^{p^{\prime}} d \sigma+\alpha\left\|\varphi_{0}\right\|_{0, p^{\prime}, S}-\int_{S}\left(y_{1}-\bar{y}\right) \varphi_{0} d s
$$

for each $\varphi_{0}$ in $L^{p^{\prime}}(S)$ and the associated solution $\varphi$ of (1.5), the problem of minimizing $J$ in $L^{p^{\prime}}(S)$ also has a unique solution $\widehat{\varphi}_{0}$. Moreover, the minimal norm control $\hat{v}$ is given by

$$
\widehat{v}(x)=-\left|\frac{\partial \hat{\varphi}}{\partial \nu}(x)\right|^{p^{\prime}-2} \frac{\partial \widehat{\varphi}}{\partial \nu}(x) \quad \text { a.e. } \quad x \in \Gamma_{\mathbf{0}}
$$


where $\hat{\varphi}$ is the solution of (1.5) associated to $\hat{\varphi}_{0}$. We also have

$$
\left\|y(\hat{v})-y_{1}\right\|_{0, p, S}=\min \left\{\alpha,\left\|y_{1}\right\|_{0, p, S}\right\}
$$

Theorem 1.1 is proved in Section 2. A unique continuation property based on the hypothesis (1.4) leads to show the existence of a control satisfying (1.1)-(1.3) by a classical density argument. This enables to construct controls of minimal $L^{p}$-norm such as in (1.7) by using a method $[\mathrm{Lil}, 2,3]$ based on the duality theory of Fenchel and Rockafellar [Ek-Te] which gives functionals like (1.8).

In an optimal control philosophy, another approach for solving (1.1)(1.3) is to minimize for some $\varepsilon>0$ the functional

$$
H(v)=\frac{1}{p} \int_{S}\left|y(v)-y_{1}\right|^{p} d s+\frac{1}{p \varepsilon} \int_{\Gamma_{0}}|v|^{p} d \sigma .
$$

This method and the method presented in Theorem 1.1 seem to present some similarities but they are actually different. We study this relationship in Section 3. More precisely, we generalize a comparison given by [De]. The extension (Theorem 3.4) evidences some equivalence between the two approaches in reflexive spaces. The cases studied in Section 2 fit into this framework, but not those of Section 4. Except for this comparison, we will not consider the optimal control method in this article.

Following this study, in Section 4 we consider the case of the $L^{p_{-}}$ approximate controllability for $1<p<\infty$ with controls of infinity minimal norm (Theorem 4.2 ) by inversing the duality argument [ $\mathrm{Fa}-\mathrm{Pu}$ $\mathrm{Zu} 3$ ]. The case of the $L^{1}$-controllability is also studied (Theorem 4.3). In this case duality is no longer valid and we use a proof by modifying the dual problem [Fa-Pu-Zur,2]. In all the cases we obtain the same kind of quasi bang-bang controls.

In Section 5 the study of the linear case leads to state the approximate controllability result in the globally Lipschitz semilinear case (Theorem 5.1) by a fixed point method [Fa-Pu-Zu3]. A counter example based on a technical argument shows that the result is not true in the superlinear case at infinity, that is a non-linearity of the type $|y|^{r-1} y$ with $r>1[\mathrm{He}]$. Nevertheless, the result could be slightly extended to a 
non-linearity between the sublinear and the superlinear comportments at infinity $[\mathrm{Fe}]$.

To complete the study, we present in Section 6 a numerical method for the linear case in $L^{2}$ based on the duality approach of the previous sections. The method has been constructed in order to have a fast update of the control in the case of changes of $\Gamma_{0}, \alpha$ or $y_{1}$ and only a small stockage is required. We observe a loss of accuracy for small values of $\alpha$ independently of the mesh, which is a typical problem in numerical approximations in control and inverse problems. The proposed numerical method is easily generalized to the $L^{p}$ case and it can be adapted to other similar control problems. The semilinear case might be treated by a fixed point procedure, but we do not follow this idea here.

\subsection{Notations}

We recall some classical definitions. For each integer $m \geq 0$ and real $p$ with $1 \leq p \leq \infty$, we recall that $W^{m, p}(\Omega)$ are the classical Sobolev spaces with norms

$$
\begin{aligned}
& \|\varphi\|_{m, p, \Omega}=\left(\sum_{|\alpha| \leq m} \int_{\Omega}\left|D^{\alpha} \varphi(x)\right|^{p} d x\right)^{1 / p} \text { for } 1 \leq p<\infty \\
& \|\varphi\|_{m, \infty, \Omega}=\max _{|\alpha| \leq m}\left(\operatorname{esssup}_{x \in \Omega}\left|D^{\alpha} \varphi(x)\right|\right) \text { for } p=\infty
\end{aligned}
$$

For $p=2$ we denote the classical Hilbert space $H^{m}(\Omega)=W^{m, 2}(\Omega)$ and we drop the subscript $p=2$ in the norms. We recall also that $W_{0}^{m, p}(\Omega)$ is the closure of $\mathcal{D}(\Omega)$ (infinitely differentiable functions with compact support in $\Omega$ ) for the norm $\|\cdot\|_{m, p, \Omega}$. For the definition of $W^{m, p}$ on the boundary and for non integral or negative values of $m$, see for example the introduction in [Gi-Ra]. We only recall here that the trace space of $W^{m, p}(\Omega)$ is $W^{m-\frac{1}{p}, p}(\Gamma)$ for $\Gamma$ regular and that the dual space of $W_{0}^{1, p}(\Omega)$ is by definition $W^{-1, p^{\prime}}(\Omega)$. We denote by $p^{\prime}$ the conjugate exponent of $p$, i.e. $1 / p+1 / p^{\prime}=1$.

Remark. Without a loss of generality, we suppose in all sections that $f=0$. Otherwise we introduce $\bar{y}$ solution of (1.6) and the results are valid by changing $y_{1}$ by $y_{1}-\bar{y}$. 


\section{$2 \quad L^{p}$-approximate controllability}

In all this section $1<p<\infty$. Section 2.1 presents the classical density argument used to show the existence of a control satisfying (1.1)-(1.3). By using the geometrical hypothesis (1.4), a unique continuation property is proved in Section 2.2 and it leads to show Theorem 2.1. In Section 2.3 a constructive approach $[\operatorname{Lil}, 2,3]$ to construct controls of minimal $L^{p}$-norm is used and Theorem 1.1 is proved.

\subsection{Classic approach}

Theorem 2.1. Assume (1.4). If $y(v)$ denotes the solution of (1.1) for $v \in L^{p}\left(\Gamma_{0}\right)$ then the set

$$
\mathcal{R}_{p}=\left\{y(v)_{/ s} \quad \text { s.t. } \quad v \in L^{p}\left(\Gamma_{0}\right)\right\}
$$

is dense in $L^{p}(S)$.

First we give a transposition sense [Li-Ma] to (1.1). Let $\varphi$ be the solution of the following problem for $h \in L^{p^{\prime}}(\Omega)$

$$
\begin{aligned}
-\Delta \varphi+a \varphi=h & \text { in } \quad \Omega \\
\varphi=0 & \text { on } \Gamma .
\end{aligned}
$$

Under the hypothesis (1.2), we know ([Ag-Do-Ni] or [Mi], Theorem 38, VI) that (2.2) has a unique solution $\varphi \in W_{0}^{1, p^{\prime}}(\Omega) \cap W^{2, p^{\prime}}(\Omega)$ and that there exists a constant $C$ which depends only on $\Omega, p$ and $a$ such that

$$
\|\varphi\|_{2, p^{\prime}, \Omega} \leq C\|h\|_{0, p^{\prime}, \Omega} .
$$

If we multiply (1.1a) by the solution $\varphi$ of (2.2) and if we integrate by parts, we obtain the following weak characterization of (1.1).

Lemma 2.2. The unique solution $y \in L^{p}(\Omega)$ of

$$
\int_{\Omega} y h d x=-\int_{\Gamma_{0}} v \frac{\partial \varphi}{\partial \nu} d \sigma \quad \forall h \in L^{p^{\prime}}(\Omega)
$$

where $\varphi$ denotes the solution of (2.2) associated to $h$, is also a solution of (1.1) (with $f=0$ ) in a weak sense and conversely. Moreover, $\Delta y \in$ $L^{p}(\Omega)$ and there exists a constant $C$ such that

$$
\|y\|_{0, p, \Omega} \leq C\|v\|_{0, p, \Gamma_{0}} .
$$


Remark. Since $\Delta y \in L^{p}(\Omega)$ then $y \in W_{l o c}^{2, p}(\Omega)$ ([Li-Ma], Theorem 3.2, p.138) and thus the trace of $y$ on $S$ makes sense if $S$ is regular and strictly included in $\Omega$.

Lemma 2.3. For each $\varphi_{0} \in L^{p^{\prime}}(S)$, there exists a unique solution $\varphi \in W^{1, p^{\prime}}(\Omega)$ of problem (1.5). Moreover, if $S$ is regular and strictly included in $\Omega$ and $\Gamma$ is regular, then

$$
\frac{\partial \varphi}{\partial \nu} \in W^{1-\frac{1}{p^{\prime}, p^{\prime}}}(\Gamma)
$$

and there exist constants $C_{1}$ and $C_{2}$ such that

$$
\left\|\frac{\partial \varphi}{\partial \nu}\right\|_{0, p^{\prime}, \Gamma} \leq C_{1}\|\varphi\|_{1, p^{\prime}, \Omega} \leq C_{2}\left\|\varphi_{0}\right\|_{0, p^{\prime}, S} .
$$

Proof. Case $p=2$. From (1.5) we have

$$
\|\nabla \varphi\|_{0, \Omega}^{2}+\int_{\Omega} a(x)|\varphi|^{2} d x=\int_{S} \varphi_{0} \varphi d s,
$$

But

$$
\begin{aligned}
\beta & =\lambda_{1}\left(1-\frac{\lambda_{1}-\beta}{\lambda_{1}}\right)=\inf _{H_{0}^{2}(\Omega)} \frac{\|\nabla \varphi\|_{0, \Omega}^{2}}{\|\varphi\|_{0, \Omega}^{2}}\left(1-\frac{\lambda_{1}-\beta}{-\lambda_{1}}\right) \\
& \leq \frac{\|\nabla \varphi\|_{0, \Omega}^{2}}{\|\varphi\|_{0, \Omega}^{2}}\left(1-\frac{\lambda_{1}-\beta}{\lambda_{1}}\right)
\end{aligned}
$$

since from the condition (1.2) we have $0<\frac{\lambda_{1}-\beta}{\lambda_{1}}<1$. Thus

$$
\frac{\lambda_{1}-\underline{\beta}_{1}}{\lambda_{1}}\|\nabla \varphi\|_{0, \Omega}^{2} \leq\|\nabla \varphi\|_{0, \Omega}^{2}-\beta\|\varphi\|_{0, \Omega}^{2} \leq\|\nabla \varphi\|_{0, \Omega}^{2}+\int_{\Omega} a(x)|\varphi|^{2} d x .
$$

Then (1.5) has a unique solution thaks to the Poincaré inequality and the Lax-Milgram Lemma. Combining this result with (2.8) and using a trace theorem we obtain the second inequality in (2.7) with $C_{2}=C \lambda_{1} /\left(\lambda_{1}-\beta\right)$.

For the general case $p \neq 2$ (see also [La-Ur] and [Ne]), let us first notice that $\delta_{S} \varphi_{0} \in W^{-1, p^{\prime}}(\Omega)$ if $\varphi_{0} \in L^{p^{\prime}}(S)$. Indeed, by definition and by using classical trace theorems we obtain 


$$
\begin{aligned}
\left\|\delta_{S} \varphi_{0}\right\|_{-1, p^{\prime}, \Omega} & =\sup \left\{\int_{S} \varphi_{0} \varphi d s ; \varphi \in W_{0}^{1, p}(\Omega),\|\varphi\|_{1, p, \Omega} \leq 1\right\} \\
& \leq \sup \left\{\left\|\varphi_{0}\right\|_{0, p^{\prime}, S}\|\varphi\|_{1-\frac{1}{p}, p, S} ; \varphi \in W_{0}^{1, p}(\Omega),\|\varphi\|_{1, p, \Omega} \leq 1\right\} \\
& \leq C\|\varphi\|_{0, p^{\prime}, S}
\end{aligned}
$$

Now, let us denote by $T h$ the solution of (2.2). We know that $T$ is an isomorphism from $L^{p}$ onto $W^{2, p} \cap W_{0}^{1, p}$ (we drop $\Omega$ to simplify the expressions). But $T=T^{*}$ is also an isomorphism from $\left(W^{2, p} \cap W_{0}^{1, p}\right)^{\prime}$ onto $L^{p^{\prime}}$. If $[A, B]$ denotes the 1/2-interpolated space between $A$ and $B$ (see [Li-Ma], Theorem 1.4) we also see that $T$ is an isomorphism between the corresponding interpolated spaces:

$$
T:\left[L^{p^{\prime}},\left(W^{2, p} \cap W_{0}^{1, p}\right)^{\prime}\right] \rightarrow\left[W^{2, p^{\prime}} \cap W_{0}^{1, p^{\prime}}, L^{p^{\prime}}\right] .
$$

But

$$
\left[L^{p^{\prime}},\left(W^{2, p} \cap W_{0}^{1, p}\right)^{\prime}\right]=\left[L^{p}, W^{2, p} \cap W_{0}^{1, p}\right]^{\prime}=\left(W_{0}^{1, p}\right)^{\prime}=W^{-1, p^{\prime}}
$$

and $\left[W^{2, p^{\prime}} \cap W_{0}^{1, p^{\prime}}, L^{p^{\prime}}\right]=W_{0}^{1, p^{\prime}}$. Therefore $T$ is an isomorphism from $W^{-1, p^{\prime}}$ onto $W_{0}^{1, p^{\prime}}$. This is equivalent to the existence and uniqueness of problem (1.5) and the second inequality in (2.7) follows from (2.9).

To prove (2.6) and the first inequality in (2.7), let $\theta \in C^{\infty}(\bar{\Omega})$ be a function such that $\theta=1$ in a neighborhood of $\Gamma$ and $\theta=0$ in a neighborhood of $S$ (this is possible since $\bar{S} \subset \Omega)$. One may note that for the solution $\varphi$ of (1.5) we have:

$$
\Delta(\theta \varphi)=\varphi \Delta \theta+2 \nabla \theta \cdot \nabla \varphi+\theta \Delta \varphi=\varphi \Delta \theta+2 \nabla \theta \cdot \nabla \varphi+a \theta \varphi
$$

and it follows that $\theta \varphi$ is the solution of

$$
\begin{aligned}
-\Delta(\theta \varphi)+a(\theta \varphi) & =h \quad \text { in } \Omega \\
\theta \varphi & =0 \text { on } \Gamma,
\end{aligned}
$$

where $h=-\varphi \Delta \theta+2 \nabla \theta \cdot \nabla \varphi \in L^{p^{\prime}}(\Omega)$. Therefore $\theta \varphi \in W_{0}^{1, p^{\prime}}(\Omega) \cap W^{2, p^{\prime}}(\Omega)$ and consequently by classical trace theorems

$$
\frac{\partial(\theta \varphi)}{\partial \nu}=\frac{\partial \varphi}{\partial \nu} \in W^{1-\frac{1}{p^{\prime}}, p^{\prime}}(\Gamma)
$$

and

$$
\left\|\frac{\partial \varphi}{\partial \nu}\right\|_{1-\frac{1}{p^{\prime}, p^{\prime}, \Gamma}}=\left\|\frac{\partial(\theta \varphi)}{\partial \nu}\right\|_{1-\frac{1}{p^{\prime}, p^{\prime}, \Gamma}} \leq C\|\theta \varphi\|_{2, p^{\prime}, \Omega} \leq\|h\|_{0, p^{\prime}, \Omega} \leq\|\varphi\|_{1, p^{\prime}, \Omega} .
$$

Finally we obtain the first inequality in (2.7) due to the continuous injection of $W^{1-\frac{1}{p}, p^{\prime}}(\Gamma)$ in $L^{p^{\prime}}(\Gamma)$. 


\subsection{Unique continuation property}

Lemma 2.4. Assume (1.4). Let $\varphi$ be the solution of problem (1.5). Then

$$
\frac{\partial \varphi}{\partial \nu}=0 \text { on } \Gamma_{0} \text { implies } \varphi=0 \text { in } \Omega
$$

Let us define the set of all the arcs which connect $S$ with $\Gamma_{0}$ as

$$
\mathcal{A}\left(S ; \Gamma_{0}\right)=\{c \in C([0,1] ; \bar{\Omega}) ; c(t) \in \Omega \backslash \bar{S} \forall t \in] 0,1\left[, c(0) \in S, c(1) \in \Gamma_{0}\right\}
$$

The geometrical condition (1.4) can be more technically stated as follows:

$$
\bar{S} \subset \Omega \text { and } \forall x \in S, \exists c \in \mathcal{A}\left(S ; \Gamma_{0}\right) \text { such that } c(0)=x \text {. }
$$

Let us also define the exterior and interior sets of $S$ relative to $\Gamma_{0}$ as

$$
\begin{aligned}
& S_{\text {ext }}=\left\{c(t) ; c \in \mathcal{A}\left(S ; \Gamma_{0}\right), t \in\right] 0,1[\} \\
& S_{i n t}=\Omega \backslash \bar{S}_{\text {ext }} .
\end{aligned}
$$

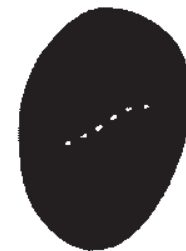

(a)

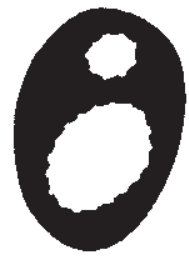

(b)

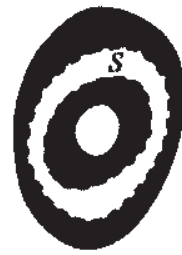

(c)

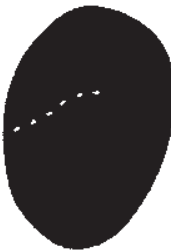

(d)

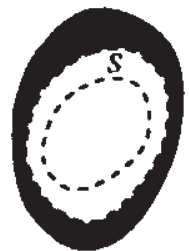

(c)

FIG. 2. In all cases $\Gamma_{0}$ is the whole boundary. The curve $S$ is represented with a dashed line. In (a), (b) and (c) the geometrical hypothesis (1.4) is satisfied but not in (d) and (e). The set $S_{e x t}$ is shaded in each case.

From the definitions above, the following geometrical properties are easily deduced.

Proposition 2.5. Assume (1.4). Then

(i) $S_{\text {ext }}$ is a non-empty open set and we have : $\bar{S} \subset \partial S_{\text {ext }}, \Gamma_{0} \subset \partial S_{\text {ext }}$. Moreover, if $S_{\text {ext }}$ has several connected components, the boundary of each of them contains a nonempty open subset of $\Gamma_{0}$.

(ii) $S_{\text {int }}$ may be empty but if not, $\partial S_{\text {int }} \subset \bar{S} \cup \Gamma$. 
Proof. (of Lemma 2.4) The solution $\varphi$ of (1.5) clearly satisfies

$$
\begin{aligned}
-\Delta \varphi+a \varphi & =0 \text { in } S_{\text {ext }} \\
\varphi & =0 \text { on } \Gamma \\
\frac{\partial \varphi}{\partial \nu} & =0 \text { on } \Gamma_{0},
\end{aligned}
$$

and it is easy to prove that $\varphi \in W_{\text {loc }}^{2, p^{\prime}}\left(S_{\text {ext }}\right)$, therefore, since $\Gamma_{0} \subset \partial S_{\text {ext }}$, by unique continuation [Sa-Sc] $\varphi=0$ in $S_{\text {ext }}$. Since $\varphi \in W^{1, p^{\prime}}(\Omega)$, we have necessarily $\varphi=0$ on $\partial S_{\text {ext }}$. As $\bar{S} \subset \partial S_{\text {ext }}$, it also vanishes on $\bar{S}$ and thus $\varphi=0$ on $\partial S_{\text {int }}$. If $S_{\text {int }}$ is not empty (otherwise $\varphi=0$ in $\Omega=S_{\text {ext }} \cup \bar{S}$ ) then

$$
\begin{aligned}
-\Delta \varphi+a \varphi & =0 \text { in } S_{i n t} \\
\varphi & =0 \text { on } \partial S_{i n t}
\end{aligned}
$$

and this implies $\varphi=0$ in $S_{\text {int }}$. Therefore, $\varphi$ vanishes in all $\Omega=S_{e \pm t} \cup S_{\text {int }} \cup \bar{S}$.

Proof. (of Theorem 2.1) Let $g \in L^{p^{\prime}}(S)$ be given and let $y(v)$ be the solution of (1.1) in the weak sense (2.4). We will show that

$$
\int_{S} y(v) g d s=0 \quad \forall v \in L^{p}\left(\Gamma_{0}\right) \text { implies } g \equiv 0 \text { on } S .
$$

If $\varphi$ is the solution of (1.5) with $\varphi_{0}=g$ then by (2.4)

$$
\int_{S} y g d s=-\int_{\Gamma_{0}} v \frac{\partial \varphi}{\partial \nu} d \sigma=0 \quad \forall v \in L^{p}\left(\Gamma_{0}\right)
$$

Therefore $\frac{\partial \varphi}{\partial \nu}=0$ and by the unique continuation property of Lemma 2.4, $\varphi \equiv 0$ in $\Omega$, so $g=\varphi_{0} \equiv 0$.

\subsection{Constructive approach}

There exists a more constructive approach to the $L^{p}$ approximate controllability on a curve which is in fact an explicit method to find controls of minimal norm. The method was introduced by J.-L. Lions (see $[\mathrm{Li1}, 2,3]$ ) and is based on the duality theory of Fenchel and Rockafellar. We use the following duality theorem.

Theorem 2.6. [Ek-Te] Let $V$ be a Banach space and $Y$ a separable topological vector space. Let $F: V \rightarrow \boldsymbol{R}$ and $G: Y \rightarrow \boldsymbol{R}$ be two convex functions. Let $L: V \rightarrow Y$ be a linear continuous operator. We suppose that there exists 
$v_{0} \in V$ such that $F\left(v_{0}\right)<+\infty, G\left(L v_{0}\right)<+\infty$ and that $G$ is continuous at $L v_{0}$. Then, if the infimum is finite, we have:

$$
\inf _{v \in V}(F(v)+G(L v))=\sup _{w^{*} \in Y^{*}}\left(-F^{*}\left(L^{*} w^{*}\right)-G^{*}\left(-w^{*}\right)\right)
$$

The supremum in (2.19) is attained at least in one point $\widehat{w}^{*} \in Y^{*}$. If the infimum is also attained at $\widehat{v} \in V$ (for example if $V$ is reflerive and $F(v)+$ $G(L v) \rightarrow+\infty$ as $\|v\| \rightarrow \infty)$, the following extremal relations are satisfied:

$$
\begin{aligned}
& F(\widehat{v})+F^{*}\left(L^{*} \widehat{w}^{*}\right)-<L^{*} \widehat{w}^{*}, \widehat{v}>=0 \\
& G(L \widehat{v})+G^{*}\left(-\widehat{w}^{*}\right)+<\widehat{w}^{*}, L \widehat{v}>=0
\end{aligned}
$$

Remark. The respective duality products between $V$ and $Y$ and their topological dual spaces $V^{*}$ and $Y^{*}$ are denoted equally by $\langle\cdot, \cdot\rangle$. The respective norms are $\|\cdot\|$ and $\|\cdot\|_{*}$. We recall that $F^{*}$ is the conjugate function of $F$ in $V$, i.e.

$$
F^{*}\left(v^{*}\right)=\sup _{v \in V}\left(\left\langle v^{*}, v>-F(v)\right) \text { for each } v^{*} \in V^{*}\right.
$$

and similarly for $G^{*}$ defined in $Y^{*}$. Moreover $L^{*}$ denotes the adjoint operator of $L$. We present in Table 1 some functions $F$ and their conjugates which are useful here. The symbol $I_{[-\alpha, \alpha]}$ denotes for $\alpha>0$ the convex function equals to 0 into the interval $[-\alpha, \alpha]$ and to $+\infty$ in its complement.

TABLE 1. Conjugate functions.

\begin{tabular}{c|c}
\hline Function $F(v)$ & Conjugate $F^{*}\left(v^{*}\right)$ \\
\hline$I_{[-\alpha, \alpha]}\left(\left\|v-v_{0}\right\|\right)$ & $\alpha\left\|v^{*}\right\|,+\left\langle v^{*}, v_{0}\right\rangle$ \\
$\frac{1}{p}\|v\|^{p}$ & $\frac{1}{p^{p}}\left\|v^{*}\right\|_{*}^{p^{\prime}}$
\end{tabular}

We will also use the following well known property (Young's inequality) which can be easily proved by developing $\log (a b)$ (see for instance $[\mathrm{Br}]$ ).

Proposition 2.7. Let $1<p<\infty, 1 / p+1 / p^{\prime}=1, a \geq 0, b \geq 0$ be given. Then

$$
\frac{1}{p} a^{p}+\frac{1}{p^{\prime}} b^{p^{\prime}}-a b \geq 0
$$

and the equality holds if and only if $a^{p}=a b=b^{p^{\prime}}$.

Proof. (of Theorem 1.1) We apply Theorem 2.6 with $V=L^{p}\left(\Gamma_{0}\right)$ and $Y=$ $L^{p}(S)$. We also take

$$
F(v)=\frac{1}{p}\|v\|_{0, p, \Gamma_{0}}^{p} \quad, \quad G(w)=I_{[-\alpha, \alpha]}\left(\left\|w-y_{1}\right\|_{0, p, s}\right) \quad, \quad L(v)=\left.y(v)\right|_{s} .
$$


We can write (1.7) in the form of the infimum in (2.13). In this case, the infimum is attained at a unique point. To see this, let us notice that the set $U_{a d}$ is not empty, since the existence of a control function that satisfies (1.3) is assured by Theorem 2.1. Moreover, it is easy to see that $U_{a d}$ is a convex set. Then, since $\frac{k}{p}\|\cdot\|_{0, p, S}^{p}$ is a strictly convex and coercive functional, there exists a unique $\widehat{v} \in L^{p}(S)$ which minimizes (1.7).

Functions $F$ and $G$ are clearly convex. By Theorem 2.1 we can chose $v_{0}$ such that

$$
\left\|y\left(v_{0}\right)-y_{1}\right\|_{0, p, S}<\alpha,
$$

then $F\left(v_{0}\right)<+\infty$ and $G\left(y\left(v_{0}\right)_{/ s}\right)=0$ and due to the continuous dependence of $y$ on $v$ (see (2.5)), there exists a neighborhood $\mathcal{O}$ of $v_{0}$ such that $G\left(y(v)_{/ s}\right)=0$ for all $v \in \mathcal{O}$, thus $G$ is continuous at $\left.y\left(v_{0}\right)\right|_{s}$. Therefore, the identity $(2.13)$ is valid in this case. We denote by $\widehat{\varphi}_{0}$ a function where the supremum is attained.

We precise the dual problem of (1.7) given by the right hand side in (2.13). From Table 1, we see that

$$
F^{*}\left(v^{*}\right)=\frac{1}{p^{\prime}}\left\|v^{*}\right\|_{0, p^{\prime}, \Gamma_{0}}^{p^{\prime}} \quad, \quad G^{*}\left(w^{*}\right)=\alpha\left\|w^{*}\right\|_{0, p^{\prime}, S}+\int_{S} y_{1} w^{*} d s,
$$

and then

$$
F^{*}\left(L^{*} w^{*}\right)+G\left(-w^{*}\right)=\frac{1}{p^{\prime}}\left\|L^{*} w^{*}\right\|_{0, p^{\prime}, \Gamma_{0}}^{p^{\prime}}+\alpha\left\|w^{*}\right\|_{0, p^{\prime}, S}-\int_{S} y_{1} w^{*} d s
$$

Let us identify $L^{*}$. From (2.4)

$$
\int_{S} y(v) \varphi_{0} d s=-\int_{\Gamma_{0}} v \frac{\partial \varphi}{\partial \nu} d \sigma
$$

and then

$$
L^{*} \varphi_{0}=-\frac{\partial \varphi}{\partial \nu}
$$

We obtain from (2.15) and (2.16) the expression (1.8) for $J$ (here $\bar{y}=0$ ).

In our case, the extremal relation (2:14a) can be written as

$$
\frac{1}{p}\|\hat{v}\|_{0, p, \Gamma_{\mathbf{0}}}^{p}+\frac{1}{p^{\prime}}\left\|L^{*} \hat{\varphi}_{0}\right\|_{0, p^{\prime}, \Gamma_{\mathbf{0}}}^{p^{\prime}}-\int_{\Gamma_{\mathbf{0}}} \hat{v} L^{*} \hat{\varphi}_{0} d \sigma=0
$$

then

$$
\int_{\Gamma_{0}}\left(\frac{1}{p}|\widehat{p}(x)|^{p}+\frac{1}{p^{\prime}}\left|L^{*} \hat{\varphi}_{0}(x)\right|^{p^{\prime}}-\widehat{v}(x) L^{*} \hat{\varphi}_{0}(x)\right) d \sigma=0 .
$$


From Proposition 2.7 the integrand of the last expression is non negative thus

$$
|\widehat{v}(x)|^{p}=\widehat{v}(x) L^{*} \hat{\varphi}_{0}(x)=\left|L^{*} \widehat{\varphi}_{0}(x)\right|^{p^{\prime}} \text { a.e. } x \in \Gamma_{0},
$$

and multiplying by $L^{*} \widehat{\varphi}_{0}(x)$ we obtain the relation (1.9). To see that $\hat{\varphi}_{0}$ is unique, we use the fact that $\widehat{v}$ is unique. Indeed, if $\hat{\varphi}_{0}^{1}$ and $\hat{\varphi}_{0}^{2}$ are two functions where the minimum of $J$ is attained, then from the first equality in (2.18)

$$
L^{*}\left(\widehat{\varphi}_{0}^{1}-\widehat{\varphi}_{0}^{2}\right)=0 \text {. }
$$

Thus by unique continuation (Lemma 2.4), the corresponding solutions of (1.5) satisfy $\hat{\varphi}^{1}=\widehat{\varphi}^{2}$ and therefore $\hat{\varphi}_{0}^{1}=\widehat{\varphi}_{0}^{2}$.

On the other hand, since we know that $\left\|L \hat{v}-y_{1}\right\|_{0, p, S} \leq \alpha$, the extremal relation $(2.14 \mathrm{~b})$ gives

$$
\alpha\left\|\hat{\varphi}_{0}\right\|_{0, p^{\prime}, S}-\int_{S} y_{1} \hat{\varphi}_{0} d s+\int_{S} L \hat{v} \hat{\varphi}_{0} d s=0 .
$$

Thus

$$
\alpha\left\|\hat{\varphi}_{0}\right\|_{0, p^{\prime}, S}=-\int_{S}\left(L \hat{v}-y_{1}\right) \widehat{\varphi}_{0} d s \leq\left\|L \hat{v}-y_{1}\right\|_{0, p, S}\left\|\hat{\varphi}_{0}\right\|_{0, p^{\prime}, S}
$$

from which, if $\widehat{\varphi}_{0} \neq 0$

$$
\left\|L \hat{v}-y_{1}\right\|_{Q, p, S}=\alpha .
$$

$\operatorname{By}(1.9) \widehat{\varphi}_{0}=0$ implies $\hat{v}=0$ and then $\left\|L \hat{v}-y_{1}\right\|_{0, p, s}=\left\|y_{1}\right\|_{0, p}, s \leq \alpha$. Conversely, if $\left\|y_{1}\right\|_{0, p, s} \leq \alpha$ then $J\left(\varphi_{0}\right) \geq 0$ for all $\varphi_{0} \in L^{p^{\prime}}(S)$ and $J(0)=0$, by uniqueness we obtain that $\widehat{\varphi}_{0}=0$. This proves $(1.10)$.

Remark. In the case $p=2$ and $\alpha<\left\|y_{1}\right\|_{0, S}$ a more precise result than (1.10) is

$$
\left(y(\hat{v})-y_{1}\right)(x)=-\alpha \frac{\hat{\varphi}_{0}(x)}{\left\|\widehat{\varphi}_{0}^{-}\right\|_{\hat{\theta}, p^{\prime}, S}^{-}} \quad \text { a.e. } \quad x \in S .
$$

Indeed, if we use (2.27) and (2.28) we have

$$
\begin{aligned}
\left\|\alpha \frac{\widehat{\varphi}_{0}}{\left\|\hat{\varphi}_{0}\right\|_{0, S}}+\left(L \hat{v}-y_{1}\right)\right\|_{0, S}^{2} & =\alpha^{2}+\alpha^{2}+2 \alpha \frac{1}{\left\|\widehat{\varphi}_{0}\right\|_{0, S}} \int_{S} \hat{\varphi}_{0} \cdot\left(L \widehat{v}-y_{1}\right) d s \\
& =2 \alpha^{2}-2 \alpha^{2}=0 .
\end{aligned}
$$

In fact, the property of uniqueness of the minima and other properties of $J$ can be proved independently, as we see in the following result. 
Lemma 2.9. Assume (1.4). For each $\alpha>0$ and $y_{1} \in L^{p}(S)$ the functional $J$ defined in (1.8) is continuous, strictly convex and

$$
\liminf _{\left\|\varphi_{0}\right\|_{0, p^{\prime}, s} \rightarrow \infty} \frac{J\left(\varphi_{0}\right)}{\left\|\varphi_{0}\right\|_{0, p^{\prime}, s}} \geq \alpha
$$

Moreover, the function $\hat{\varphi}_{0} \in L^{p^{\prime}}(S)$ where $J$ achieves its unique minimum satisfies

$$
\widehat{\varphi}_{0} \neq 0 \Leftrightarrow \alpha<\left\|y_{1}\right\|_{0, p, s}
$$

Proof. We give a proof such as in [Fa-Pu-Zu3]. To see that $J$ is continuous, we only need to recall the continuity property (2.7). $\mathrm{J}$ is strictly convex since it is the sum of strictly convex terms.

To prove (2.22), we take a sequence such that $\left\|\varphi_{0}^{n}\right\|_{0, p^{\prime}, S} \rightarrow \infty$ and we denote by $\varphi_{\mathrm{n}}$ the solution of (1.5) associated to $\varphi_{0}^{\mathrm{n}}$. If we define

$$
\tilde{\varphi}_{n}=\frac{\varphi_{n}}{\left\|\varphi_{0}^{n}\right\|_{0, p^{\prime}, S}} \quad \text { and } \quad \tilde{\varphi}_{0}^{n}=\frac{\varphi_{0}^{n}}{\left\|\varphi_{0}^{n}\right\|_{0, p^{\prime}, S}},
$$

then by (2.7) $\left\|\varphi_{n}\right\|_{1, p^{\prime}, \Omega} \leq C\left\|\varphi_{0}^{n}\right\|_{0, p^{\prime}, S}$, and thus $\tilde{\varphi}_{n}$ is bounded in $W^{1, p^{\prime}}(\Omega)$ and $\widetilde{\varphi}_{0}^{n}$ is also bounded in $L^{p^{\prime}}(S)$. As a result, up to a subsequence:

$$
\begin{aligned}
& \widetilde{\varphi}_{n} \rightarrow \widetilde{\varphi} \text { in } W^{1, p^{\prime}}(\Omega) \text {-weakly } \\
& \widetilde{\varphi}_{0}^{n} \rightarrow \widetilde{\varphi}_{0} \text { in } L^{p^{\prime}}(S) \text {-weakly. }
\end{aligned}
$$

But

$$
\frac{J\left(\varphi_{0}^{n}\right)}{\left\|\varphi_{0}^{n}\right\|_{0, p^{\prime}, S}}=\frac{1}{p^{\prime}}\left\|\varphi_{0}^{n}\right\|_{0, p^{\prime}, S}^{p^{\prime}-1} \int_{\Gamma_{0}}\left|\frac{\partial \tilde{\varphi}_{n}}{\partial \nu}\right|^{p^{\prime}} d \sigma-\int_{S} y_{1} \tilde{\varphi}_{0}^{n} d s+\alpha
$$

If we define the number:

$$
\gamma=\liminf _{n} \frac{1}{p^{\prime}} \int_{\Gamma_{\mathbf{0}}}\left|\frac{\partial \widetilde{\varphi}_{n}}{\partial \nu}\right|^{p^{\prime}} d \sigma
$$

we have two cases: if $\gamma>0$, since $\left\|\varphi_{0}^{n}\right\|_{0, p^{\prime}, S} \rightarrow \infty$ the equality (2.24) implies that

$$
\liminf _{n} \frac{J\left(\varphi_{0}^{n}\right)}{\left\|\varphi_{0}^{n}\right\|_{0, p^{\prime}, S}} \rightarrow+\infty>\alpha .
$$

We consider now the case $\gamma=0$. By using the continuity and the convexity of $\|\cdot\|_{0, p^{\prime}, \Gamma_{\mathbf{0}}}^{p^{\prime}}$ and (2.7) we can deduce that

$$
\frac{1}{p^{\prime}} \int_{\Gamma_{0}}\left|\frac{\partial \widetilde{\varphi}}{\partial \nu}\right|^{p^{\prime}} d \sigma \leq \liminf _{n} \frac{1}{p^{\prime}} \int_{\Gamma_{0}}\left|\frac{\partial \widetilde{\varphi}_{n}}{\partial \nu}\right|^{p^{\prime}} d \sigma=\gamma=0,
$$


then $\frac{\partial \tilde{\varphi}}{\partial \nu}=0$ on $\Gamma_{0}$ and by Lemma 2.4 we see that $\tilde{\varphi}=0$ in $\Omega$. Therefore

$$
\tilde{\varphi}_{n} \rightarrow 0 \text { in } W^{1, p^{\prime}}(\Omega) \text {-weakly }
$$

and all the sequence converges. But by definition $\widetilde{\varphi}_{n}$ is the solution of (1.5) for $\widetilde{\varphi}_{0}^{n}$, and by continuity (see (2.7)) $\tilde{\varphi}=0$ is the solution of (1.5) for $\widetilde{\varphi}_{0}$ and then $\widetilde{\varphi}_{0}=0$. Then

$$
\widetilde{\varphi}_{0}^{n} \rightarrow 0 \text { in } L^{p^{\prime}}\left(S^{\prime}\right) \text {-weakly. }
$$

Using this fact, from (2.24) we obtain

$$
\liminf _{n} \frac{J\left(\varphi_{0}^{n}\right)}{\left\|\vec{\varphi}_{0}^{*}\right\|_{0, p^{\prime}, S}^{-}}=\frac{1}{p^{\prime}} \liminf _{n}\left\|\varphi_{0}^{n}\right\|_{0, p^{\prime}, S}^{p^{\prime}-1} \int_{\Gamma_{0}}\left|\frac{\partial \tilde{\varphi}_{n}}{\partial \nu}\right|^{p^{\prime}} d \sigma-0+\alpha \geq \alpha,
$$

and (2.22) is proved. The direct implication in (2.23) is easy. For the other one, let us suppose that $\widehat{\varphi}_{0}=0$. Then

$$
0 \leq \lim _{t \rightarrow 0^{+}} \frac{J\left(t \varphi_{0}\right)}{t}=\alpha\left\|\varphi_{0}\right\|_{0, p^{\prime}, S}-\int_{S} y_{1} \varphi_{0} d s \quad \forall \varphi_{0} \in L^{p^{\prime}}(S) .
$$

Taking $\varphi_{0}=y_{1}^{p / p^{\prime}}$ we obtain $\alpha\left\|y_{1}\right\|_{0, p, S}^{p-1}-\left\|y_{1}\right\|_{0, p, S}^{p} \geq 0$ and then $\left\|y_{1}\right\|_{0, p, S} \leq \alpha$.

\section{Relationship with the optimal control theory}

We provide here a generalization of a result given in [De], see also [Ca-Gl-Li].

Let $E$ be a separable topological vector space and $F$ a reflexive Banach space. Let $L: F \rightarrow E$ be a linear continuous operator and $L^{*}: E^{\prime} \rightarrow F^{\prime}$ its adjoint operator. Let $\varepsilon>0$ and $\alpha>0$ be given real numbers and $y_{1} \in E$ a given function.

We consider the following abstract control problem:

Find $v \in F$ such that $\left\|L v-y_{1}\right\|_{E}$ and $\|v\|_{F}$ are small.

We introduce two different strategies or minimization problems and their respective dual problems in the sense of Theorem 2.6. Here $1<p<\infty$ and $1 / p+1 / p^{\prime}=1$. The following hypothesis ensures that all conditions about $v_{0}$ in Theorem 2.6 are fulfilled. We suppose that

$\exists v \in F \quad$ such that $\quad\left\|L v-y_{1}\right\|_{E}<\alpha$. 
Optimal control strategy: (Tychonov's regularization)

$$
\begin{aligned}
\min _{v \in F} \frac{1}{p}\|v\|_{F}^{p} & +\frac{1}{\varepsilon p^{p}}\left\|L v-y_{1}\right\|_{E}^{p}=-\min _{w \in E^{\prime}} \frac{1}{p^{\prime}}\left\|L^{*} w\right\|_{F^{\prime}}^{p^{p}} \\
& +\frac{c^{p^{\prime}}-1}{p^{\prime}}\|w\|_{E^{\prime}}^{p^{\prime}}-\left\langle y_{1}, w\right\rangle_{E, E^{\prime}} .
\end{aligned}
$$

Duality strategy: (Fenchel's regularization)

$$
\begin{aligned}
\min _{v \in F} \frac{1}{p}\|v\|_{F}^{p} & +I_{[-\alpha, \alpha]}\left\|L v-y_{1}\right\|_{E}=-\min _{w \in E^{\prime}} \frac{1}{p^{\prime}}\left\|L^{*} w\right\|_{F^{\prime}}^{p^{\prime}} \\
& +\alpha\|w\|_{E^{\prime}}-\left\langle y_{1}, w\right\rangle_{E, E^{\prime}}
\end{aligned}
$$

The extremality conditions (2.14a) and (2.14b) which characterize the solutions of (3.2) and (3.3) are respectively

$$
\begin{gathered}
\frac{1}{p}\|\hat{v}\|_{F}^{p}+\frac{1}{p^{\prime}}\left\|L^{*} \hat{w}\right\|_{F^{\prime}}^{p^{\prime}}-\left\langle\widehat{v}, L^{*} \widehat{w}\right\rangle_{F_{i} F^{\prime}}=0 \\
\frac{1}{\varepsilon p}\left\|L \widehat{v}-y_{1}\right\|_{E}^{p}+\frac{\varepsilon^{p^{\prime}-1}}{p^{\prime}}\|\widehat{w}\|_{E^{\prime}}^{p^{\prime}}-\left\langle y_{1}, \widehat{w}\right\rangle_{E, E^{\prime}}+\langle L \hat{v}, \widehat{w}\rangle_{E, E^{\prime}}=0
\end{gathered}
$$

and

$$
\begin{gathered}
\frac{1}{p}\|\tilde{v}\|_{F}^{p}+\frac{1}{p^{\prime}}\left\|L^{*} \tilde{w}\right\|_{F^{\prime}}^{p^{\prime}}-\left\langle\tilde{v}, L^{*} \tilde{w}\right\rangle_{F, F^{\prime}}=0 \\
\left\|L \tilde{v}-y_{1}\right\|_{E} \leq \alpha \text { and } \alpha\|\tilde{w}\|_{E^{\prime}}-\left\langle y_{1}, \tilde{w}\right\rangle_{E, E^{\prime}}+\langle L \tilde{v}, \widetilde{w}\rangle_{E, E^{\prime}}=0 .
\end{gathered}
$$

Lemma 3.1. For $\varepsilon>0$ given, let $(\widehat{v}, \widehat{w})$ be a solution of problem (9.2). Then we have

$$
\varepsilon\|\hat{w}\|_{E^{\prime}}=\left\|L \hat{v}-y_{1}\right\|_{E}^{p-1} .
$$

Proof. The condition (3.4b) implies that

$$
\frac{1}{p}\left\|L \hat{v}-y_{1}\right\|_{E}^{p}+\frac{1}{p^{\prime}}\|\varepsilon \hat{w}\|_{E^{\prime}}^{p^{\prime}} \leq\left\|L \hat{v}-y_{1}\right\|_{E}\|\varepsilon \hat{w}\|_{E^{\prime}}
$$

then, using Proposition 2.7 we obtain

$$
\varepsilon^{p^{\prime}}\|\widehat{w}\|_{E^{\prime}}^{p^{\prime}}=\left\|L \widehat{v}-y_{1}\right\|_{E}^{p}
$$

and since $p / p^{\prime}=p-1$ we can deduce (3.6).

Lemma 3.2. For $\alpha<\left\|y_{1}\right\|_{E}$ given, let $(\widetilde{v}, \widetilde{w})$ be a solution of (3.3). Then we have

$$
\alpha=\left\|L \bar{v}-y_{1}\right\|_{E} .
$$


Remark. The case $\alpha \geq\left\|y_{1}\right\|_{E}$ is not really interesting since in this case $v=0$ and $w=0$ is a solution of $(3.3)$.

Proof. One may note that if $\widetilde{w}=0$ then from the extremality condition (3.5a) we have $\widetilde{v}=0$ and if we use (3.5b) we see that $\left\|y_{1}\right\|_{E} \leq \alpha$. By hypothesis $\alpha<\left\|y_{1}\right\|_{E}$ and then here $\widetilde{w} \neq 0$. Now from (3.5b) we see that

$$
\alpha\|\tilde{w}\|_{E^{\prime}} \leq\left\|L \tilde{v}-y_{1}\right\|_{E}\|\tilde{w}\|_{E^{\prime}}
$$

and so we have (3.7).

We directly have from these two Lemmas the following Corollary.

Corollary 3.3. For $\varepsilon>0$ and $\alpha<\left\|y_{1}\right\|_{E}$ given, let $(v, w)$ be a solution of (3.2) and (3.3) at the same time, then we necessarily have

$$
\alpha^{p-1}=\varepsilon\|w\|_{E^{\prime}} .
$$

The converse property is also true in the following sense.

\section{Theorem 3.4.}

(i) For $\varepsilon>0$ given, let $(\hat{v}, \widehat{w})$ be a solution of problem (3.2). Then $(\widehat{v}, \hat{w})$ is also a solution of (3.3) for

$$
\widehat{\alpha}=e^{p^{\prime}-1}\|\widehat{w}\|_{E^{\prime}}^{p^{\prime}-1} .
$$

(ii) For $\alpha<\left\|y_{1}\right\|_{E}$ given, let ( $\left.\tilde{v}, \widetilde{w}\right)$ be a solution of (3.3). Then ( $\left.\widetilde{v}, \widetilde{w}\right)$ is also a solution of (3.2) for

$$
\widetilde{\varepsilon}=\alpha^{p-1}\|\tilde{w}\|_{E^{\prime}}^{-1} .
$$

Proof. To prove (i), let $\varepsilon>0$ be given and let $(\widehat{v}, \widehat{w})$ be a solution of problem (3.2). Take $\hat{\alpha}$ such as in (3.8). Then, using the relation (3.6) we easily see that

$$
\frac{1}{\varepsilon p}\left\|L \hat{v}-y_{1}\right\|_{E}^{p}+\frac{\varepsilon^{p^{\prime}-1}}{p^{\prime}}\|\widehat{w}\|_{E^{\prime}}^{p^{\prime}}=\widehat{\alpha}\|\widehat{w}\|_{E^{\prime}}
$$

and

$$
\left\|L \widehat{v}-y_{1}\right\|_{E}^{p}=\hat{\alpha} .
$$


From these two latter expressions, if we compare the extremality conditions (3.6) and (3.7), it is clear that $(\hat{v}, \hat{w})$ also is a solution of (3.6) and then for (3.3) for $\alpha=\hat{\alpha}$.

The proof of (ii) is completely similar. If $\alpha<\left\|y_{1}\right\|_{E}$ is given and ( $\left.\tilde{v}, \tilde{w}\right)$ is a solution of (3.3), by choosing $\widetilde{\varepsilon}$ such as in (3.9) and by using the relation (3.7) we can deduce that

$$
\frac{1}{\widetilde{\varepsilon} p}\left\|L \widetilde{v}-y_{1}\right\|_{E}^{p}+\frac{\widetilde{\varepsilon}^{p^{\prime}-1}}{p^{\prime}}\|\widetilde{w}\|_{E^{\prime}}^{p^{\prime}}=\alpha\|\widetilde{w}\|_{E^{\prime}}
$$

If we compare again the extremality conditions (3.6) and (3.7) again, it is easy to see that $(\widetilde{v}, \widetilde{w})$ is a solution of (3.7) and then it is a solution of (3.2) with $\varepsilon=\widetilde{\varepsilon}$.

\section{First extension: quasi bang-bang controls}

\section{1 $L^{p}$-controllability with an $L^{\infty}$-minimal norm control}

We give an extension of Theorem 2.8 to controls of minimal infinity norm. Here $1<p \leq q<\infty$.

Definition 4.1. We say that $v \in \varphi \operatorname{sgn} \psi$ if $v(x)=\varphi(x) \frac{\psi(x)}{\psi(x) \mid}$ a.e. in the set $\{x \mid \psi(x) \neq 0\}$ and $|v(x)| \leq|\varphi(x)|$ a.e. on the set $\{x \mid \psi(x)=0\}$.

Theorem 4.2. Assume (1.4). For each $y_{1} \in L^{p}(S)$ and $\alpha>0$ there exists a control $v \in L^{\infty}\left(\Gamma_{0}\right)$ such that the solution $y(v)$ of (1.1) satisfies (1.3). Moreover, if $\mathcal{U}_{a d}^{\infty}$ represents the set of all control functions in $L^{\infty}\left(\Gamma_{0}\right)$ which satisfies (1.3), then the minimization problem:

$$
\min _{v \in \mathcal{U}_{a \triangleleft}^{\infty} \infty} \frac{1}{q}\|v\|_{0, \infty, \Gamma_{0}}^{q}
$$

has a solution $\widehat{v}$. The minimization problem in $L^{p^{\prime}}(S)$ for

$$
J\left(\varphi_{0}\right)=\frac{1}{q^{\prime}}\left\|\frac{\partial \varphi}{\partial \nu}\right\|_{0,1, \Gamma_{0}}^{q^{\prime}}+\alpha\left\|\varphi_{0}\right\|_{0, p^{\prime}, S}-\int_{S} y_{1} \varphi_{0} d s
$$

where $\varphi$ represents the solution of (1.5) for each $\varphi_{0} \in L^{p^{\prime}}(S)$, has a unique solution $\widehat{\varphi}_{0}$ and $\widehat{v}$ satisfies on $\Gamma_{0}$

$$
\widehat{v} \in-\left\|\frac{\partial \hat{\varphi}}{\partial \nu}\right\|_{0,1, \Gamma_{0}}^{q^{\prime}-1} \operatorname{sgn} \frac{\partial \hat{\varphi}}{\partial \nu}
$$


where $\hat{\varphi}$ is the solution of (1.5) for $\widehat{\varphi}_{0}$. For the error we also have the identity $(1.10)$.

Proof. The proof is also based on Theorem 2.6, but here we take the minimization of (4.2) as the primal problem. We chose $Y=L^{1}\left(\Gamma_{0}\right), V=L^{p^{\prime}}(S)$,

$$
F\left(\varphi_{0}\right)=\alpha\left\|\varphi_{0}\right\|_{0, p^{\prime}, S}-\int_{S} y_{1} \varphi_{0} d s, G(w)=\frac{1}{q^{\prime}}\|w\|_{0,1, \Gamma_{0}}^{q^{\prime}}, L \varphi_{0}=-\left.\frac{\partial \varphi}{\partial \nu}\right|_{\Gamma_{0}} .
$$

We have $F(0)=0<\infty, G(0)=0<\infty, G$ is clearly continuous at 0 and $J=F+G \circ L$. We notice that $J$ also satisfies the coercive property (2.22) with the same proof of Lemma 2.9 (we only use the continuity and convexity of $\left.\|\cdot\|_{0,1, \Gamma_{0}}^{q^{\prime}}\right)$. Since here $V$ is reflexive, the minimization problem for $J$ has a unique solution $\hat{\varphi}_{0}$ and its dual problem following Theorem 2.6 is

$$
-\min _{v \in L^{\infty}\left(\Gamma_{0}\right)}\left(I_{[-\alpha, \alpha]}\left(|| L^{*} v-y_{1} \|_{0, p, S}\right)+\frac{1}{q}\|v\|_{0, \infty, \Gamma_{0}}^{q}\right)
$$

equivalent to (4.1) since $L^{*} \varphi_{0}=y(v)_{/ s}$. Theorem 2.6 enables us to see that the extremality conditions (2.14a) and (2.14b) hold. The error property (1.10) is deduced directly from (2.14a) and (4.3) is obtained from the other extremal relation $(2.14 \mathrm{~b})$ as follows:

$$
\begin{aligned}
& 0=\frac{1}{q^{\prime}}\left\|\frac{\partial \hat{\varphi}}{\partial \nu}\right\|_{0,1, \Gamma_{0}}^{q^{\prime}}+\frac{1}{q}\|\widehat{v}\|_{0, \infty, \Gamma_{0}}^{q}-\int_{\Gamma_{0}} \hat{v} \frac{\partial \hat{\varphi}}{\partial \nu} d \sigma \\
& \geq \int_{\Gamma_{0}} \frac{\partial \hat{\varphi}}{\partial \nu} \mid d \sigma\|\hat{v}\|_{0, \infty, \Gamma_{0}}-\int_{\Gamma_{0}} \widehat{v} \frac{\partial \widehat{\varphi}}{\partial \nu} d \sigma \geq 0
\end{aligned}
$$

then from Proposition 2.7

$$
\|\hat{v}\|_{0, \infty, \Gamma_{0}}^{q}=\|\hat{v}\|_{0, \infty, \Gamma_{0}}\left\|\frac{\partial \hat{\varphi}}{\partial \nu}\right\|_{0,1, \Gamma_{0}}=\left\|\frac{\partial \hat{\varphi}}{\partial \nu}\right\|_{0,1, \Gamma_{0}}^{q^{\prime}} .
$$

Also

$$
\int_{\Gamma_{0}}\left(\left|\frac{\partial \hat{\varphi}}{\partial \nu}(x)\right|\|\widehat{v}\|_{0, \infty, \Gamma_{0}}-\frac{\partial \hat{\varphi}}{\partial \nu}(x) \widehat{v}(x)\right) d \sigma=0
$$

thus

$$
\frac{\partial \widehat{\varphi}}{\partial \nu}(x) \widehat{v}(x)=\left|\frac{\partial \widehat{\varphi}}{\partial \nu}(x)\right|\|\widehat{v}\|_{0, \infty, \Gamma_{0}} \quad \text { a.e. } \quad x \in \Gamma_{0} .
$$

But (4.4) means

$$
|\widehat{v}(\boldsymbol{x})| \leq\left\|\frac{\partial \hat{\varphi}}{\partial \nu}\right\|_{0,1, \Gamma_{0}}^{q^{\prime}-1} \quad \text { a.e. } \quad x \in \Gamma_{0}
$$

and then (4.3) holds. 
Remark. With the same proof of Lemma 2.9, we can prove the equivalence (2.23) in the case of Theorem 4.2. So the case $\alpha \geq\left\|y_{1}\right\|_{0, p, S}$ implies $\hat{\varphi}_{0}=0$ and $\widehat{v}=0$ (unique).

Remark. The expression (4.3) means that the control $\hat{v}$ is quasi bang-bang. If $\alpha<\left\|y_{1}\right\|_{0, p_{1} s}$ then $\hat{v}$ is not unique in general. In fact, all solutions of (4.1) only differ on the set $\left\{x \in \Gamma_{0} \mid \frac{\partial \hat{\varphi}}{\partial \nu}(x)=0\right\}$. The measurement of this set is an open problem, but it does not contain any non-empty open set of $\Gamma_{0}$ due to the unique continuation property.

\section{2 $L^{1}$-controllability with an $L^{\infty}$-minimal norm control}

In the present case, we can not apply a duality argument. However, the idea is to directly introduce a functional like (4.2) and to write the extremality condition at its minimum to obtain a control which satisfies the error condition (4.7). Here we consider the solution $\varphi$ of (1.5) with $\varphi_{0} \in L^{\infty}(S)$. The function $\varphi$ is regular near $\Gamma_{0}$ and its normal derivative on $\Gamma_{0}$ satisfies

$$
\left\|\frac{\partial \varphi}{\partial \nu}\right\|_{0,1, \Gamma_{0}} \leq C\left\|\varphi_{0}\right\|_{0, \infty, S} .
$$

Given $y_{1} \in L^{1}(S)$ and $\alpha>0$, we define for $1<q<\infty$

$$
J\left(\varphi_{0}\right)=\frac{1}{q}\left\|\frac{\partial \varphi}{\partial \nu}\right\|_{0,1, \Gamma_{0}}^{q}+\alpha\left\|\varphi_{0}\right\|_{0, \infty, S}+\int_{S} y_{1} \varphi_{0} d s .
$$

We can prove the analogous of the coercive property (2.22) for this new functional using the weak-* topology in $L^{\infty}$. As a result, $J$ has a unique minimum and we can extend the result of Section 4.1 in a weaker form. Here $1<q<\infty$.

Theorem 4.3. Assume (1.4). For all $y_{1} \in L^{1}(S)$ and $\alpha>0$, if $\hat{\varphi}$ is the solution of (1.5) associated with the minimum $\hat{\varphi}_{0}$ in $L^{\infty}(S)$ of (4.5), then there exists

$$
\widehat{v} \in-\left\|\frac{\partial \hat{\varphi}}{\partial \nu}\right\|_{0,1, \Gamma_{0}}^{q-1} \operatorname{sgn} \frac{\partial \hat{\varphi}}{\partial \nu} \quad \text { on } \Gamma_{0}
$$

such that the solution $y(\hat{v})$ of (1.1) satisfies

$$
\left\|y(\widehat{v})-y_{1}\right\|_{0,1, s} \leq \alpha .
$$

Remark. A priori, (4.7) is not valid for all $\widehat{v}$ satisfying (4.6), but this is true if the set $\left\{x \in \Gamma_{0} \mid \frac{\partial \widehat{\varphi}}{\partial \nu}(x)=0\right\}$ has zero measure, since in this case $\widehat{v}$ is unique (a.e.). 
Proof. If $J$ attains its minimum at $\hat{\varphi}_{0}$, we have for each $\phi_{0} \in L^{\infty}(S)$ that $J\left(\hat{\varphi}_{0}+\varepsilon \phi_{0}\right)-J\left(\hat{\varphi}_{0}\right) \geq 0$ for all $\varepsilon>0$. We note $J_{1}\left(\hat{\varphi}_{0}\right)=\frac{1}{q}\left\|\frac{\partial \hat{\varphi}}{\partial \nu}\right\|_{0,1, \Gamma_{0}}^{q}$, where $\hat{\varphi}$ is the solution of (1.5). By using the triangular inequality we obtain that

$$
\frac{1}{\varepsilon}\left(J_{1}\left(\hat{\varphi}_{0}+\varepsilon \phi_{0}\right)-J_{1}\left(\hat{\varphi}_{0}\right)\right)+\alpha\left\|\phi_{0}\right\|_{0, \infty, S}-\int_{S} y_{1} \phi_{0} d s \geq 0 \quad \forall \phi_{0} \in L^{\infty}(S) .
$$

One may observe that $J_{1}$ is subdifferentiable, then by taking limit as $\varepsilon \rightarrow 0$ we can deduce that there exists $\xi \in \partial J_{1}\left(\widehat{\varphi}_{0}\right)$ such that

$$
\int_{S} \xi \phi_{0} d s+\alpha\left\|\phi_{0}\right\|_{0, \infty, S}-\int_{S} y_{1} \phi_{0} d s \geq 0 \quad \forall \phi_{0} \in L^{\infty}(S) .
$$

But $J_{1}=\bar{J}_{1} \circ L$ where $\bar{J}_{1}(u)=\frac{1}{q}\|u\|_{0,1, \Gamma_{0}}^{q}$ and $L \varphi_{0}=-\frac{\partial \varphi}{\partial \nu}$ over $\Gamma_{0}$. Since $\partial\left(\bar{J}_{1} \circ L\right)\left(\hat{\varphi}_{0}\right)=L^{*} \partial \bar{J}_{1}\left(\hat{\varphi}_{0}\right)$ (see [Ek-Te] p. 27) we can deduce that $\xi$ is of the form

$$
\xi=L^{*} \hat{v}
$$

where $\hat{v}$ satisfies (see $[\mathrm{Ek}-\mathrm{Te}]$ p.21)

$$
\frac{1}{q}\left\|\frac{\partial \hat{\varphi}}{\partial \nu}\right\|_{0,1, \Gamma_{0}}^{q}+\frac{1}{q^{\prime}}\|\hat{v}\|_{0, \infty}^{q^{\prime}, \Gamma_{0}}=\int_{\Gamma_{0}} \frac{\partial \hat{\varphi}}{\partial \nu} \widehat{v} d \sigma
$$

and this implies (4.6) with the sarne proof as in Theorem 4.2. Here we also have $L^{*} v=y(\widehat{v})$, where $y(\hat{v})$ is the solution of (1.1). By using (4.9) and if we follow (4.8) again, we obtain

$$
\int_{S} y(\widehat{v}) \phi_{0} d s+\alpha\left\|\phi_{0}\right\|_{0, \infty, S}-\int_{S} y_{1} \phi_{0} d s \geq 0 \quad \forall \phi_{0} \in L^{\infty}(S) .
$$

This implies that

$$
\int_{S}\left(y(\hat{v})-y_{1}\right) \phi_{0} d s \geq-\alpha\left\|\phi_{0}\right\|_{0, \infty, S} \quad \forall \phi_{0} \in L^{\infty}(S),
$$

and taking $\phi_{0} \in-\operatorname{sgn}\left(y(\widehat{v})-y_{1}\right)$ we obtain (4.7).

Remark. The equivalence (2.23) is also valid in this case and takes the same proof of Lemma 2.9 with $\varphi_{0} \in \operatorname{sgn} y_{1}$ at the end. Consequently, the case $\alpha \geq\left\|y_{1}\right\|_{0,1, S}$ implies $\hat{v}=0$ in Theorem 4.3. 


\section{Second extension: the semilinear Laplace equa- tion}

\subsection{Main Result}

The following theorem states the $L^{2}$-approximate controllability of the semilinear Laplace equation. The proof of the $L^{P}(1 \leq p<\infty)$ case is similar and we do not give the details (see [Fa-Pu-Zu3]).

Theorem 5.1. Let $f$ be a real valued and continuous function and let us suppose that there exist positive constants $\beta$ and $\gamma$ such that

$$
-\lambda_{1}<-\beta \leq \frac{f(s)-f(0)}{s} \leq \gamma \text { for all } s \in R \backslash\{0\}
$$

where $\lambda_{1}>0$ denotes the smallest eigenvalue of the $-\Delta$ operator in $\Omega$ with Dirichlet homogeneous boundary conditions. If we consider the problem :

$$
\begin{array}{rll}
-\Delta y+f(y)=0 & \text { in } & \Omega \\
y=0 & \text { on } \Gamma \backslash \Gamma_{0} \\
y=v & \text { on } \Gamma_{0}
\end{array}
$$

then, under the geometrical hypothesis (1.4), the set

$$
\mathcal{R}_{2}=\left\{y(v) / s \quad \text { s.t. } \quad v \in L^{2}\left(\Gamma_{0}\right)\right\}
$$

is dense in $L^{2}(S)$.

Proof. Let $y_{1} \in L^{2}(S)$ and $\alpha>0$ be given. We will prove that there exists a control function $v \in L^{2}\left(\Gamma_{0}\right)$ such that

$$
\left\|y(v)_{/ s}-y_{1}\right\|_{0, s} \leq \alpha .
$$

Without loss of generality, we can suppose that $f(0)=0$. We also suppose that $f \in C^{1}(\boldsymbol{R})$ (see the Remark at the end of the proof). The role of $f$ will be played by the the following real function:

$$
g(s)=\frac{f(s)}{s} \text { if } s \neq 0 \text { and } g(0)=f^{\prime}(0) .
$$

For each $z \in L^{2}(\Omega)$ fixed, we associate the solution $y(z, v)$ of

$$
\begin{aligned}
-\Delta y+g(z) y=0 & \text { in } \Omega \\
y=0 & \text { on } \Gamma \backslash \Gamma_{0} \\
y=v & \text { on } \Gamma_{0} .
\end{aligned}
$$


It is easy to deduce from (5.1) that $g$ satisfies the conditions (1.2). Then, thanks to Theorem 2.1, we can choose $v(z) \in L^{2}\left(\Gamma_{0}\right)$ such that $\left\|y(z, v(z))-y_{1}\right\|_{0, S} \leq$ $\alpha$. Among these possible controls, we choose $v(z)$ with minimal $L^{2}$-norm. A characterization of such control is given by duality in Theorem 1.1. We recall that if we consider the solution $\varphi\left(z, \varphi_{0}\right)$ of

$$
\begin{aligned}
-\Delta \varphi+g(z) \varphi & =\delta_{S} \varphi_{0} \text { in } \Omega \\
\varphi & =0 \text { on } \Gamma
\end{aligned}
$$

and we define as $\widehat{\varphi}_{0}(z)$ the minimum in $L^{2}(S)$ of the functional

$$
J_{z}\left(\varphi_{0}\right)=\frac{1}{2} \int_{\Gamma_{0}}\left|\frac{\partial \varphi\left(z, \varphi_{0}\right)}{\partial \nu}\right|^{2} d \sigma+\alpha\left\|\varphi_{0}\right\|_{0, s}-\int_{S} y_{1} \varphi_{0} d s
$$

then

$$
v(z)=-\frac{\partial \varphi\left(z, \hat{\varphi}_{0}(z)\right)}{\partial \nu} \text { on } \Gamma_{0} .
$$

The idea is to show that the application $\mathcal{A}: z \rightarrow y(z, v(z))$ has a fixed point $\bar{z}$ in $L^{2}(\Omega)$. In this case $g(\bar{z}) \bar{y}=f(\bar{y})$, where $\bar{y}=y(\bar{z}, v(\bar{z}))$ and, as a consequence, $v(\bar{z})$ is the required control function for which (5.3) holds.

Let us show that the application $\mathcal{A}: z \rightarrow y(z, v(z))$ is continuous from $L^{2}(\Omega)$ into itself. We take a sequence $z_{n} \rightarrow z$ in $L^{2}(\Omega)$, and for a fixed $\varphi_{0} \in L^{2}(S)$ we denote the solution of (5.4) by $\varphi_{n}=\varphi\left(z_{n}, \varphi_{0}\right)$. By using Lemma 2.3 we obtain $\left\|\varphi_{n}\right\|_{1, \Omega} \leq C\left\|\varphi_{0}\right\|_{0, s}$. Consequently, up to a sub-sequence, there exists a function $\varphi \in H_{0}^{1}(\Omega)$ such that

$$
\varphi_{n} \rightarrow \varphi \text { in } H_{0}^{1}(\Omega) \text {-weakly. }
$$

Let us notice that

$$
\begin{aligned}
\left\|g\left(z_{n}\right) \varphi_{n}-g(z) \varphi\right\|_{0, \Omega} & \leq\left\|g\left(z_{n}\right) \varphi_{n}-g(z) \varphi_{n}\right\|_{0, \Omega}+\left\|g(z) \varphi_{n}-g(z) \varphi\right\|_{0, \Omega} \\
& \leq\left\|\varphi_{n}\right\|_{0, \Omega}\left\|g\left(z_{n}\right)-g(z)\right\|_{0, \Omega}+\|g(z)\|_{0, \Omega}\left\|\varphi_{n}-\varphi\right\|_{0, \Omega}
\end{aligned}
$$

thus

$$
g\left(z_{n}\right) \varphi_{n} \rightarrow g(z) \varphi \text { in } L^{2}(\Omega) \text {-strongly. }
$$

In order to identify $\varphi$, remark that by definition $\varphi_{n}$ is solution of

$$
\int_{\Omega} \nabla \varphi_{n} \cdot \nabla \phi d x+\int_{\Omega} g\left(z_{n}\right) \varphi_{n} \phi d x=\int_{S} \varphi_{0} \phi d s \text { for all } \phi \in H_{0}^{1}(\Omega) \text {. }
$$

By taking limit as $n \rightarrow \infty$, using (5.8) and (5.9) and the fact that $\left\langle\Delta \varphi_{n}, \phi\right\rangle$ converges to $\langle\Delta \varphi, \phi\rangle$ for all $\phi \in \mathcal{D}(\Omega)$, we obtain

$$
\int_{\Omega} \nabla \varphi \cdot \nabla \phi d x+\int_{\Omega} g(z) \varphi \phi d x=\int_{S} \varphi_{0} \phi d s \text { for all } \phi \in H_{0}^{1}(\Omega)
$$


which implies that

$$
\varphi=\varphi\left(z, \varphi_{0}\right) .
$$

In fact, the convergence is strong in (5.8). Indeed, if we take $\phi=\varphi_{n}$ and $\phi=\varphi$ in (5.10) and (5.11) respectively, we obtain

$$
\begin{gathered}
\left\|\nabla \varphi_{n}\right\|_{0, \Omega}^{2}+\int_{\Omega} g\left(z_{n}\right)\left|\varphi_{n}\right|^{2} d x=\int_{S} \varphi_{0} \varphi_{n} d s \\
\|\nabla \varphi\|_{0, \Omega}^{2}+\int_{\Omega} g(z)|\varphi|^{2} d x=\int_{S} \varphi_{0} \varphi d s,
\end{gathered}
$$

and by taking limit as $n \rightarrow \infty$ in (5.13) and by comparing this limit with (5.14) we can see that $\nabla \varphi_{n}$ converges to $\nabla \varphi$ in $L^{2}(\Omega)^{N}$. To sum up, we see that if $\varphi_{0}$ is fixed then

$$
\varphi\left(z_{n}, \varphi_{0}\right) \rightarrow \varphi\left(z, \varphi_{0}\right) \quad \text { in } \quad H_{0}^{1}(\Omega) \text {-strongly. }
$$

The same argument is also valid to show that

$$
\varphi\left(z_{n}, \hat{\varphi}_{0}\left(z_{n}\right)\right) \rightarrow \varphi\left(z, \hat{\varphi}_{0}(z)\right) \text { in } H_{0}^{1}(\Omega) \text {-weakly, }
$$

provided that

$$
\widehat{\varphi}_{0}\left(z_{n}\right) \rightarrow \widehat{\varphi}_{0}(z) \text { in } L^{2}(\Omega) \text {-weakly. }
$$

By contradiction, suppose that there exists a sub-sequence such that $\left\|\widehat{\varphi}_{0}\left(z_{n}\right)\right\|_{0, S} \rightarrow \infty$. Since $\hat{\varphi}_{0}\left(z_{n}\right)$ minimizes the functional $J_{z_{n}}$ then

$$
J_{z_{n}}\left(\hat{\varphi}_{0}\left(z_{n}\right)\right) \leq J_{z_{n}}\left(\varphi_{0}\right) \text { for all } \varphi_{0} \in L^{2}(S) \text {. }
$$

From (5.15) and Lemma 2.3, the normal derivative of $\varphi\left(z_{n}, \varphi_{0}\right)$ also converges in $L^{2}\left(\Gamma_{0}\right)$ to the normal derivative of $\varphi\left(z, \varphi_{0}\right)$, so $J_{z_{n}}\left(\varphi_{0}\right)$ converges to $J_{z}\left(\varphi_{0}\right)$. Therefore, there exists a constant $M$ such that

$$
J_{z_{n}}\left(\widehat{\varphi}_{0}\left(z_{n}\right)\right) \leq M
$$

The same proof such as in Lemma 2.9 shows that for each $z_{n}, J_{z_{n}}$ is a continuous and strictly convex functional and that

$$
\liminf _{\left\|\widehat{\varphi}_{0}\left(z_{n}\right)\right\|_{0, S} \rightarrow \infty} \frac{J_{z_{n}}\left(\widehat{\varphi}_{0}\left(z_{n}\right)\right)}{\left\|\widehat{\varphi}_{0}\left(z_{n}\right)\right\|_{0, S}} \geq \alpha
$$

As a result, the uniform bound in (5.18) is a contradiction. Then, up to a sub sequence, $\widehat{\varphi}_{0}\left(z_{n}\right)$ weakly converges in $L^{2}(S)$ to a limit denoted $\widehat{\varphi}_{0}$, that is

$$
\widehat{\varphi}_{0}\left(z_{n}\right) \rightarrow \widehat{\varphi}_{0} \text { in } L^{2}(S) \text {-weakly. }
$$


Now, we must prove that $\widehat{\varphi}_{0}=\widehat{\varphi}_{0}(z)$. Indeed, by $(5.19)$ and using the fact that $\hat{\varphi}_{0}\left(z_{n}\right)$ minimizes $J_{z_{n}}$ we obtain

$J_{z}\left(\hat{\varphi}_{0}\right) \leq \liminf _{n} J_{z_{n}}\left(\hat{\varphi}_{0}\left(z_{n}\right)\right) \leq \lim _{n} \inf J_{z_{n}}\left(\varphi_{0}\right)=J_{z}\left(\varphi_{0}\right)$ for all $\varphi_{0} \in L^{2}(S)$,

therefore $\widehat{\varphi}_{0}$ minimizes $J_{z}$ and $\widehat{\varphi}_{0}=\widehat{\varphi}_{0}(z)$. Consequently, the convergences (5.17) and (5.16) are valid. From the definition (5.7), by using (5.16) and Lemma 2.3 we see that the control function $v\left(z_{n}\right)$ weakly converges to $v(z)$ in $L^{2}\left(\Gamma_{0}\right)$. Finally, since $z \rightarrow z_{n}$ in $L^{2}(\Omega)$, it is easy to show that $y\left(z_{n}, v\left(z_{n}\right)\right)$ strongly converges to $y(z, v(z))$ in $L^{2}(\Omega)$. Therefore $\mathcal{A}$ is a continuous map.

Let us prove that the application $\mathcal{A}: z \rightarrow y(z, v(z))$ maps $L^{2}(\Omega)$ in a bounded subset of $L^{2}(\Omega)$. We have $\|g(z)\|_{0, \Omega}$ uniformly bounded in $z$. Thanks to Lemma 2.3 we see that $\left\|\varphi\left(z, \varphi_{0}\right)\right\|_{1, \Omega}$ and the $L^{2}$-norm of its normal derivative remain uniformly bounded in $z$, therefore $J_{z}\left(\varphi_{0}\right)$ is bounded with respect to $z$. Consequently, there exists a constant $M$ only depending on $\varphi_{0}$, such that $J_{z}\left(\hat{\varphi}_{0}(z)\right) \leq M$. If there existed a subsequence such that $\left\|\hat{\varphi}_{0}\left(z_{n}\right)\right\|_{0,5} \rightarrow \infty$ we would necessarily have

$$
\alpha \leq \frac{J_{z_{n}}\left(\hat{\varphi}_{0}\left(z_{n}\right)\right)}{\left\|\hat{\varphi}_{0}\left(z_{n}\right)\right\| \|_{0, S}^{-}} \leq \frac{J_{z_{n}}\left(\varphi_{0}\right)}{\left\|\hat{\varphi}_{0}\left(z_{n}^{-}\right)\right\|_{0, S}} \leq \frac{M}{\left\|\hat{\varphi}_{0}\left(z_{n}\right)\right\|_{0, S}} \rightarrow 0
$$

and this is impossible. Therefore $\left\|\hat{\varphi}_{0}(z)\right\|_{0, S}$ is uniformly bounded in $z$. From Lernma 2.3 we have $\varphi\left(z, \hat{\varphi}_{0}(z)\right)$ uniformly bounded in $H_{0}^{1}(\Omega)$ and then $v(z)$ defined in (5.7) is also uniformly bounded. We conclude that $y(z, v(z))$ remains bounded in $L^{2}(\Omega)$, uniformly with respect to $z$.

Remark. In the proof we suppose $f \in C^{1}(\Omega)$. We can always take a regularizing sequence

$$
f_{n}=\rho_{n} * f, \quad \operatorname{supp} p_{n} \subset[-1 / n, 1 / n]
$$

which satisfies (5.1) for each $n$ (see [Fa-Pu-Zu3], Proposition 3.4). Thus we obtain a control $v_{n}$ satisfying (5.3). Taking limit as $n \rightarrow \infty$, we can pass to the limit in (5.2) and the weak limit of $v_{n}$ is the required control to assure (5.3).

\subsection{A counterexample}

We consider a non-linearity of the form:

$$
f(s)=|s|^{r-1} s \text { for } \quad r>1 \text {. }
$$

We will prove that if $y$ is solution of $(5.2)$ then the set

$$
u=\left\{y(v)_{/ s} \quad \text { s.t. } \quad v \in L^{2}\left(\Gamma_{0}\right)\right\}
$$


is bounded in $L^{2}(\Omega)$. In particular $U$ is not dense in $L^{2}(\Omega)$. The proof is based on the classic example of Bamberger [He]. If we multiply (5.2a) by $\theta y, \theta \geq 0$, $\theta \in H^{1}(\Omega) \cap L^{\infty}(\Omega)$, and if we integrate by parts we obtain

$$
\int_{\Omega} \nabla y \cdot \nabla(\theta y) d x-\int_{\Gamma_{0}} \frac{\partial y}{\partial \nu} \theta v d \sigma+\int_{\Omega} \theta f(y) y d x=0
$$

thus, if we define $q=r-1$

$$
\int_{\Omega} \theta|y|^{2} d x+\int_{\Omega} y \nabla y \cdot \nabla \theta d x+\int_{\Omega} \theta|y|^{q+2} d x-\int_{\Gamma_{0}} \frac{\partial y}{\partial \nu} \theta v d \sigma=0 .
$$

But $\frac{1}{2}+\frac{q}{2(q+2)}+\frac{1}{q+2}=1$ and $\frac{1}{2}-\frac{q+4}{2(q+2)}+\frac{1}{q+2}=0$ then by Hölder inequality

$$
\begin{aligned}
\left|\int_{\Omega} y \nabla y \cdot \nabla \theta d x\right| & \leq\left(\int_{\Omega} \theta|\nabla y|^{2} d x\right)^{\frac{1}{2}}\left(\int_{\Omega} \theta^{-\frac{q+4}{q}}|\nabla \theta|^{\frac{2(q+2)}{q}} d x\right)^{\frac{q}{2(q+2)}}\left(\int_{\Omega} \theta|y|^{q+2} d x\right)^{\frac{q}{q+2}} \\
\leq & \frac{1}{2} \int_{\Omega} \theta|\nabla y|^{2} d x+\frac{q}{2(q+2)} \int_{\Omega} \theta^{-\frac{q+1}{q}}|\nabla \theta|^{\frac{2(q+2)}{q}} d x+\frac{1}{q+2} \int_{\Omega} \theta|y|^{q+2} d x
\end{aligned}
$$

therefore by (5.21)

$$
\int_{\Omega} \theta|y|^{2} d x \leq \int_{\Gamma_{0}} \frac{\partial y}{\partial \nu} \theta v d \sigma+\frac{q}{\underline{2(q+2)}} \int_{\Omega} \theta^{-\frac{2+1}{q}}|\nabla \theta|^{\frac{3(q+2)}{q}} d x .
$$

By hypothesis $S \cap \partial \Omega=\phi$, thus let $\left\{\overline{B\left(x_{i}, 2 r_{i}\right)} \subset \Omega \text { s.t. } r_{i}>0\right\}_{i=1}^{M}$ be a finite covering of the curve $S$, where $B\left(x_{i}, 2 r_{i}\right)$ denotes the ball of center $x_{i}$ and radius $2 r_{i}$. We define

$$
B_{i}=B\left(x_{i}, r_{i}\right)
$$

Applying the trace theorem for each $B_{i}$, we obtain:

$$
\|y\|_{0, S}^{2} \leq \sum_{i=1}^{M}\|y\|_{0, S \cap B_{2}}^{2} \leq \sum_{i=1}^{M} C_{i}\|y\|_{1, B_{1}}^{2},
$$

where $C_{i}, i=1, \ldots, M$ are constants which depend on $S \cap B_{i}$ and $B_{i}$.

We note that for a parameter $\gamma>0$, if $\rho=|x|$ then

$$
\int_{B_{i}}|\nabla y|^{2} d x \leq \int_{B\left(x_{i}, 2 r_{i}\right)}\left(\frac{2 r_{i}-\rho}{r_{i}}\right)^{\gamma}|\nabla y|^{2} d x
$$

Now we choose in (5.22) the function $\theta=\theta_{i}$, where

$$
\theta_{i}(x)=\left\{\begin{array}{cc}
\left(\frac{2 r_{i}-x}{r_{i}}\right)^{\gamma} & x \in B\left(x_{i}, 2 r_{i}\right) \\
0 & x \in \Omega \backslash B\left(x_{i}, 2 r_{i}\right)
\end{array},\right.
$$


thus, using (5.23) we obtain

$$
\int_{B_{i}}|\nabla y|^{2} d x \leq \gamma^{\frac{2(q+2)}{q}} \frac{2 \pi q}{2(q+2)} \int_{0}^{2 r_{i}} \rho\left(\frac{2 r_{i}-\rho}{r_{i}}\right)^{-\gamma \frac{(q+1)}{q}+2(\gamma-1) \frac{(q+2)}{q}} d \sigma
$$

and the right side integral converges if and only if the exponent is greater than -2 , that is if $\gamma>4 / q$. Here we use the fact that $q$ has been supposed strictly positive. Thus let us choose $\gamma$ such that

$$
\int_{B_{i}}|\nabla y|^{2} d x \leq C_{i}^{\prime}
$$

We conclude from (5.23) and (5.25) that $\|y\|_{0 s}<C$, where $C$ is a constant which does not depend on the control $v$. We conclude that $U$ is bounded and therefore $U$ is not dense in $L^{2}(\Omega)$.

\section{Numerical method}

\subsection{Discrete method}

Let us consider a triangulation $\tau_{h}$ of $\Omega$ where $h$ is a discretization parameter, and let us denote by $\Gamma_{h}, \Gamma_{0 h}$ and $S_{h}$ the respective discretizations of $\Gamma_{2}, \Gamma_{0}$ and $S$ induced by $\tau_{h}$. In our implementation, we suppose that the triangulation consists of triangles $K$ :

$$
\bar{\Omega}_{h}=\bigcup_{K \in \tau_{h}} K
$$

We suppose that $\Gamma_{0 h}$ is formed by $Q-1$ segments $\left[p_{i}, p_{i+1}\right], S_{h}$ is formed by $M-1$ segments $\left[q_{i}, q_{i+1}\right]$ and that each segment is a side of a triangle in $\tau_{h}$. Therefore, $Q$ and $M$ are the total number of vertices in $\Gamma_{o h}$ and $S_{h}$ respectively. We will denote by $N V$ the total number of interior vertices in $\Omega_{h}$.

We consider a finite dimensional subspace $L_{h}$ of $L^{2}\left(S_{h}\right)$, endowed with the inner product and norm of $L^{2}\left(S_{h}\right)$. The dimension of $L_{h}$ is a function of $M$ denoted $D(M)$. Then $L_{h}$ is generated by a basis $\left\{\phi_{k}\right\}_{k=1}^{D(M)}$

$$
L_{h}=\left\langle\phi_{1}, \ldots, \phi_{D(M)}\right\rangle \text {. }
$$

Let us denote by

$$
T: \varphi_{0} \in L^{2}(S) \rightarrow \varphi \in H_{0}^{1}(\Omega)
$$


the linear operator which associates $\varphi_{0}$ to $\varphi$ by (1.5), i.e., the solution of the variational problem:

$$
\int_{\Omega} \nabla \varphi \cdot \nabla \phi d x+\int_{\Omega} a \varphi \phi d x=\int_{S} \varphi_{0} \phi d s \quad \forall \phi \in H_{0}^{1}(\Omega) .
$$

If we consider a finite dimensional space $V_{0 h}$ of $H_{0}^{1}(\Omega)$, then we can define the following discrete approximation of $T$ :

$$
T_{h}: \varphi_{0 h} \in L_{h} \rightarrow \varphi_{h} \in V_{0 h}
$$

where $\varphi_{h}$ is the solution of

$$
\int_{\Omega_{h}} \nabla \varphi_{h} \cdot \nabla \phi d x+\int_{\Omega_{h}} a \varphi_{h} \phi d x=\int_{S_{h}} \varphi_{0 h} \phi d s \quad \forall \phi \in V_{0 h} .
$$

If we define the matrices

$$
\begin{gathered}
G_{k l}=\int_{\Gamma_{0 h}} \frac{\partial T_{h} \phi_{k}}{\partial t} \frac{\partial T_{h} \phi_{l}}{\partial \downarrow} d \sigma \\
C_{k l}=\int_{S_{h}} \phi_{k} \phi_{l} d s,
\end{gathered}
$$

and using that for each $\varphi_{0 h} \in L_{h}$ we have the decomposition

$$
\varphi_{0 k}=\sum_{k=1}^{D(\boldsymbol{M})} \beta_{k} \phi_{k},
$$

we can express the following quantities in terms of $\beta=\left(\beta_{1}, \ldots, \beta_{D(M)}\right)^{t}$ (a repeated index indicates summation)

$$
\begin{aligned}
& \frac{1}{2} \int_{\Gamma_{0 h}}\left|\frac{\partial T_{h} \varphi_{0 h}}{\partial \nu}\right|^{2} d \sigma=\frac{1}{2} \beta_{k} \beta_{l} \int_{\Gamma_{0 h}} \frac{\partial T_{h} \phi_{k}}{\partial \nu} \underline{\frac{\partial T_{h} \phi_{l}}{\partial \nu}} d \sigma=\frac{1}{2} \beta^{t} G \beta \\
& \left\|\varphi_{0 h}\right\|_{L_{n}}^{2}=\int_{S_{h}} \beta_{k} \phi_{k} \beta_{l} \phi_{l} d s=\beta_{k} \beta_{l} \int_{S_{\mathrm{h}}} \phi_{k} \phi_{t} d s=\beta^{t} C \beta .
\end{aligned}
$$

Besides, if we suppose that the projection $y_{1 h}$ of $y_{1}$ on $L_{h}$ is of the form $y_{1 h}=$ $f_{1 k} \phi_{k}$, we have

$$
\int_{S_{h}} y_{1 h} \varphi_{0 h} d s=\beta_{k} f_{1 l} \int_{S_{h}} \phi_{k} \phi_{l} d s=\beta^{t} C f_{1} .
$$

Therefore, we find the following finite dimensional forms of the functional $J$ and its differential $J^{\prime}$ defined for $\beta \neq 0$ :

$$
\begin{aligned}
J_{h}(\beta) & =\frac{1}{2} \beta^{t} G \beta+\alpha\left(\beta^{t} C \beta\right)^{1 / 2}-\beta^{t} C f_{1} \\
J_{h}{ }^{\prime}(\beta) & =G \beta+\alpha\left(\beta^{t} C \beta\right)^{-1 / 2} C \beta-C f_{1} \quad(\beta \neq 0) .
\end{aligned}
$$


If $\widehat{\beta}=\left(\widehat{\beta}_{1}, \ldots, \widehat{\beta}_{D(M)}\right)$ is the solution of

$$
J_{h}(\widehat{\beta})=\min _{\beta \in \boldsymbol{R}^{D(M)}} J_{h}(\beta)
$$

and if $\widehat{\varphi}_{0 h}=\sum_{k} \widehat{\beta}_{k} \phi_{k}$, the discrete control $\widehat{v}_{h}$ on $\Gamma_{0 h}$ is given by

$$
\widehat{v}_{h}=-\frac{\partial T_{h} \widehat{\varphi}_{\partial h}}{\partial D}=-\sum_{k=1}^{D(M)} \widehat{\beta}_{k} \frac{\partial T_{h} \phi_{k}}{\partial D} .
$$

\subsubsection{Operator $T_{h}$}

We introduce the standard finite element space of polynomials of degree less than or equal to 1 , which are piecewise continuous

$$
V_{h}=\left\{\varphi \in C^{0}(\bar{\Omega})|\varphi|_{T} \in P_{1}(K), \forall K \in \tau_{h}\right\}
$$

and we choose $V_{0 h}=V_{h} \cap H_{0}^{1}(\Omega)$. We denote by $\left\{\omega_{i}\right\}_{i=1}^{N}$ the canonical finite element basis in $V_{0 h}$. Now, we define the matrix $A$ and for each $\phi_{k}$ of $(6.1)$ the vector $b\left(\phi_{k}\right)$ by

$$
\begin{gathered}
A_{i j}=\int_{\Omega_{h}} \nabla \omega_{i} \cdot \nabla \omega_{j} d x+\int_{\Omega_{h}} a w_{i} w_{j} d x \\
b_{j}\left(\phi_{k}\right)=\int_{S_{h}} \phi_{k} \omega_{j} d s .
\end{gathered}
$$

From (6.5) we obtain for $k=1, \ldots, D(M)$

$$
T_{h} \phi_{k}=\sum_{i=1}^{N V} \alpha_{i} \omega_{i} \quad \text { where } A \alpha=b\left(\phi_{k}\right) .
$$

\subsubsection{Normal derivatives}

To calculate the normal derivatives in (6.6a), we multiply (1.5) by $\psi \in H^{1}(\Omega)$ and after integrating by parts we obtain

$$
\int_{\Gamma} \frac{\partial \varphi}{\partial \nu} \psi d s=-\int_{\Omega} \nabla \varphi \cdot \nabla \psi d x+\int_{\Omega} a \varphi \psi d x+\int_{S} \varphi_{0} \psi d x
$$

We recall that $\left\{p_{k}\right\}_{k=1}^{Q}$ is the set of vertices in $\Gamma_{0 h}$ and we denote by $\left\{\psi_{k}\right\}_{k=1}^{Q}$ the canonical finite element basis in $V_{h}$ associated to these vertices, i.e. $\psi_{j}\left(p_{k}\right)=$ 
$\delta_{j k}$. If we use now a Simpson quadrature formula to compute the left hand side in the equation above, we see that

$$
\sum_{k=1}^{Q} \gamma_{k} \frac{\partial \varphi}{\partial \nu}\left(p_{k}\right) \psi_{j}\left(n_{k}\right)=-\int_{\Omega_{h}} \nabla \varphi \cdot \nabla \psi_{j} d x+\int_{\Omega_{h}} a \varphi \psi_{j} d x
$$

where $\gamma_{k}$ are specific weights. We supposed that the mesh is sufficiently refined to separate the curve from the supports of the functions $\psi_{k}$. Therefore we obtain for $k=1, \ldots, Q$ the formula

$$
\frac{\partial \varphi}{\partial \nu}\left(p_{k}\right)=-\frac{1}{\gamma_{k}}\left(\int_{\Omega_{h}} \nabla \varphi \cdot \nabla \psi_{k} d x+\int_{\Omega_{h}} a \varphi \psi_{k} d x\right)
$$

See for example [Gl-Li-Li] and [Gl-Li-Tr] for a discussion about this and other possible methods to calculate the normal derivatives.

\subsubsection{Basis in $L_{h}$}

We have discretized $S_{h}$ in $M-1$ segments $I_{i}=\left[q_{i}, q_{i+1}\right]$ of length $l_{i}$ which are sides of triangles of $\tau_{h}$. We tested two different discretizations of $L^{2}\left(S_{h}\right)$, the piecewise constant functions on each segment and the continuous functions which are piecewise degree $\leq 1$ polynomials respectively:

$$
\begin{aligned}
& L_{h}^{(0)}=\left\{\phi \in L^{2}\left(S_{h}\right)|\phi|_{I_{i}} \in P_{0}, i=1, \ldots, M-1\right\} \\
& L_{h}^{(1)}=\left\{\phi \in L^{2}\left(S_{h}\right)|\phi|_{I_{1}} \in P_{1}, i=1, \ldots, M-1\right\} .
\end{aligned}
$$

We have $\operatorname{dim} L_{h}^{(0)}=M-1$ and $\operatorname{dim} L_{h}^{(1)}=2(M-1)$. We can choose orthogonal basis in this spaces, for example, with the following functions:

$$
\phi_{1}^{(0)}=\left\{\begin{array}{ll}
1 & x \in I_{i} \\
0 & \text { otherwise }
\end{array} \quad \text { and } \phi_{i}^{(1)}= \begin{cases}1-\frac{2}{l_{i}}\left(x-q_{1}\right) & x \in I_{i} \\
0 & \text { otherwise }\end{cases}\right.
$$

Then

$$
L_{h}^{(0)}=\left\langle\phi_{1}^{(0)}, \ldots, \phi_{M-1}^{(0)}\right\rangle, L_{h}^{(1)}=\left\langle\phi_{1}^{(0)}, \ldots, \phi_{M-1}^{(0)}, \phi_{1}^{(1)}, \ldots, \phi_{M-1}^{(1)}\right\rangle
$$

and it is easy to see that

$$
\int_{S_{n}} \phi_{i}^{(0)} \phi_{j}^{(0)} d s=l_{i} \delta_{i j}, \quad \int_{S_{n}} \phi_{i}^{(1)} \phi_{j}^{(1)} d s=\frac{l_{i}}{3} \delta_{i j}, \quad \int_{S_{h}} \phi_{i}^{(0)} \phi_{j}^{(1)} d s=0 .
$$

Therefore, the diagonal $C$ matrices associated to $L_{h}^{(0)}$ and $L_{h}^{(1)}$ are respectively

$$
C^{(0)}=\operatorname{diag}\left(l_{1}, \ldots, l_{M-1}\right) \quad, \quad C^{(1)}=\operatorname{diag}\left(l_{1}, \ldots, l_{M-1}, l_{1} / 3, \ldots, l_{M-1} / 3\right) .
$$




\subsection{Test examples}

We solve the $L^{2}$-approximate controllability problem (1.1)-(1.3) for $N=2$ with $f=0$ and $a=1$. Using the expression (6.13), we compute the matrices $G$ and $C$ in (6.6), then we minimize the functional (6.8) and we compute the control by using the identity $(6.11)$.

We will use three different geometries (see Figure 5), all of them satisfying the geometrical hypothesis (1.4). We work with the pararneters $\alpha, y_{1 h}$ and $\Gamma_{0 h}$. Table 2 summarizes the implementation of the discrete method applied to these examples and shows the added dependency of each stage on the principa: parameters of the problem.

TABLE 2. The numerical implementation.

\begin{tabular}{c|cc}
\hline Stage & Applied method & Dependency on \\
\cline { 2 - 2 } $\begin{array}{c}\text { Mesh and elemen- } \\
\text { tary matrices } \\
\text { System } \\
N V \times N V\end{array}$ & $\begin{array}{c}P_{1} \text {-finite elements } \\
\text { Choleski with D(M) } \\
\text { right sides } \\
\begin{array}{c}\text { Normal } \\
\text { derivatives } \\
\text { Minimization } \\
\text { and Control }\end{array}\end{array}$ & $\Omega_{h}$ \\
$\begin{array}{c}\text { Variational-quadrature } \\
\text { algorithm }\end{array}$ & $S_{h}$ \\
Quasi-Newton BFGS & $\alpha, y_{1 h}$
\end{tabular}

For the mesh and the computation of the elementary matrices we use a standard finite element package. The system $N V \times N V$ shown in (6.13) with the $D(M)$ right sides is solved by a Choleski decomposition.

In order to obtain the matrix $G$, defined in (6.6a), we use (6.13) and the variational and quadrature methods explained in Section 6.1.2 to calculate the normal derivative on the boundary. In practice, $G$ is an ill-conditioned matrix. To overcome this problem, it is necessary to work with double precision variables and to do, for example, the following preconditioning:

$$
\beta=\operatorname{diag}\left(G_{i \dot{i}}^{-1 / 2}\right) \beta^{\prime} .
$$

Matrix $C$ defined in (6.6b) is computed following the directives of Section 6.1.3 to have a sparse profile. We observe in our calculations that in the case $L_{h}=L_{h}^{(0)}$ (see (6.14)) the condition number of $G$ (normalized with (6.15)) is better than in the case $L_{h}=L_{h}^{(1)}$. This result could mean that the basis $\left\{\phi_{k}\right\}_{k=1}^{D(M)}$ must not have superfiuous elements. Therefore, we choose $C=C^{(0)}$ in all our calculations.

To solve the minimizing problem we use the variant Broyden-Fletcher-Goldfarb-Shanno (BFGS) algorithm [Pr-Te]. We make some modifications of 
this method according to (6.15) and we choose the initial guest parallel to $f_{1}$. This amounts to minimizing $J_{h}\left(\lambda f_{1}\right)$ with respect to $\lambda \in \boldsymbol{R}$. We easily obtain

$$
\beta_{0}=\left(f_{1}^{t} C f_{1}\right)^{\frac{1}{2}} \frac{\left(\left(f_{1}^{t} C f_{1}\right)^{\frac{1}{2}}-\alpha\right)}{f_{1}^{t} G f_{1}} f_{1} .
$$

The stop test is a "small" gradient or a limit of the precision machine.

Figure 5 shows three examples of controls computed with the described method. On the left column, we show the curve $S_{h}$ (dashed line) and $\Gamma_{0 h}$ (solid line). Example 1: $(N V=1287, M=20, Q=30)$ a square domain with a rectilinear curve $S$ near the upper side $\Gamma_{0}$. Example 2: $(N V=991, M=20$, $Q=110$ ) a square domain with a circular curve, Here $\Gamma_{0}$ are the left, right and upper sides. Example 3: $(N V=1983, M=30, Q=90)$ a curved domain and a central curve. $\Gamma_{0}$ is one half of the boundary.

In all the examples of Figure 5, the aim is to obtain a minimal norm control function $\widehat{v}_{h}$ such that the solution $y$ of (1.1) satisfies $y\left(\widehat{v}_{h}\right) \approx y_{1 h}=1$ on $S_{h}$. In the right column, we represent schematically the control $\widehat{v}_{h}$ by straight lines following the normal on $\Gamma_{0 h}$. The associated solution $y_{h}\left(\widehat{v}_{h}\right)$ is indicated with gray levels in the range $(-2.00,2.00)$ in all cases. Level $1.00 \pm 0.03$ is marked out in white.

Figure 4 shows two other examples with the same geometry as Example 1, but a different $y_{1 h}$. In Example $4 y_{1 h}$ is a sinusoidal function. In Example 5 $y_{1 h}$ a Heaveside function. The trace on $S_{h}$ of the calculated solution $y_{h}\left(\widehat{v}_{h}\right)$ of (1.1) (solid line) and the desired function $y_{1 h}$ (dashed line) are shown.

The total computation time in Example 3 was approximately $60 \mathrm{sec}$ in a work station HP9000. The percent of the total computation time in this example for each stage was: mesh and elementary matrices $33.0 \%$, system $50.0 \%$, normal derivatives $13.3 \%$, minimization and control $3.3 \%$. The situation is similar for the other examples.

To solve the system, the memory storage is of order $(N V+D(M)) \times N V$, but in practice $A$ is a sparse matrix. In the other stages, we must store the normal derivatives on $\Gamma, G, f_{1}$ and $C$, i.e. of order $(Q+D(M)) \times D(M)$.

The precision is studied by introducing the following errors: the theoretical error $\left\|y-y_{1}\right\|_{L^{2}(S)}=\min \left\{\alpha,\left\|y_{1}\right\|_{0, S}\right\}$ and the numerical error $\left\|y_{h}-y_{1 h}\right\|_{0, S_{h}}$, where $y_{h}$ is the solution of a discretized version of (1.1) for the computed control $\widehat{v}_{h}$. Let us notice that here there is an added error, due to this new 
discretization. We work with relative errors, i.e. the errors are multiplied by $100 /\left\|y_{1 h}\right\|_{0, s_{n}}$.

In all our examples we observe a similar relation between the numerical and theoretical errors. Figure 3 shows this relation for the initial mesh of Example I $(N V=1287, M=20, Q=30)$ and two other refinements with the same geometry. Refinement 1: $(N V=2552, M=30, Q=40)$ more elements everywhere. Refinement 2: $(N V=2063, M=20, Q=100)$ more elements near $\Gamma_{0}$.

We observe that the numerical method does not improve the precision under a minimal value of alpha ( $1 \%$ in Figure 3 ). Below this value, the numerical relative error does not decrease as expected theoretically. It is not only a discretization problem, since we observe that the more the mesh is refined, the worse is the conditioning of the minimization problem. We can see in Figure 3 that the situation is not as good as we would expect after the refinements. This is a classical situation in the numerical algorithms for control and inverse problems.

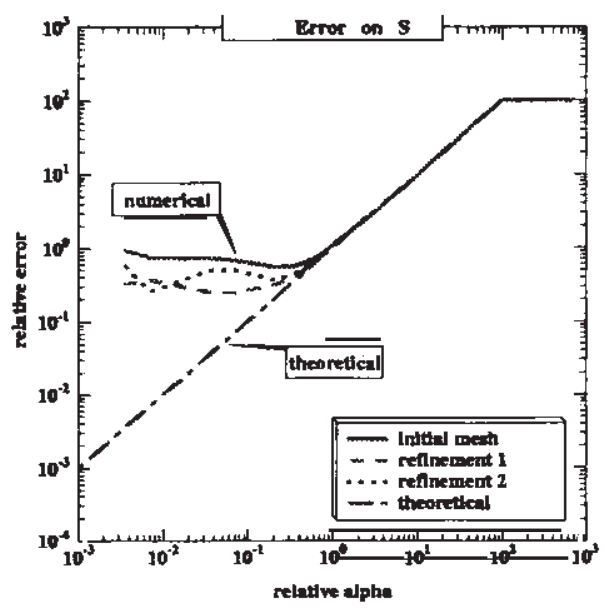

FIG. 3. Comparison between the theoretical and numerical errors.

In Examples 1, 2 and 3, the minimal numerical relative errors are $0.8 \%$, $0.3 \%$ and $6 \%$ respectively: In Examples 4 and 5 , they are $1.6 \%$ and $29 \%$ respectively. The important error in the last case is fully justified: we can not obtain a discontinuous function due to the smooth effect of the Laplace operator.

The time required to update the control represents only $3.3 \%$ of the total CPU time in the case that $y_{1 h}$ or $\alpha$ are modified (for instance only $2 \mathrm{sec}$ in 
Example 3). The stockage required in this case is small as well. It is due to the fact that we have to repeat only the minimization stage $(6.10)$, which is uncoupled to the resolution of the whole finite element system (6.13). The size of the minimization problem is only related with the total number of discretization nodes of the curve $D(M)$. This fact reduces notably the degrees of freedom and consequently the stockage and computation time.

Finally, we would like to emphasize the fact that the presented numerical implementation can easily be adapted to other similar linear control problems in which the dimension of the discretized observed space is small compared with the dimension of the discretized state space.
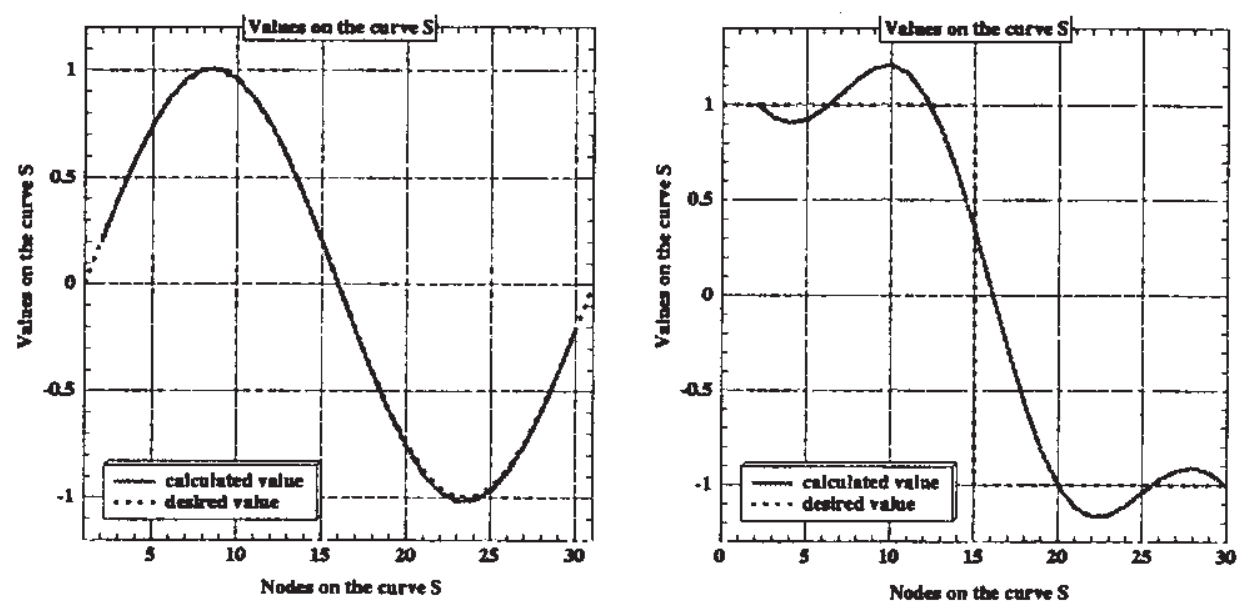

FIG, 4. Controlled solution $y_{h}\left(\widehat{v}_{h}\right)$ (solid line) and desired value $y_{1 h}$ (dashed line) on $S_{h}$. Example 4 (top): $y_{1 h}$ sinusoidal function. Example 5 (bottom): $y_{1 h}$ Heaveside function. 

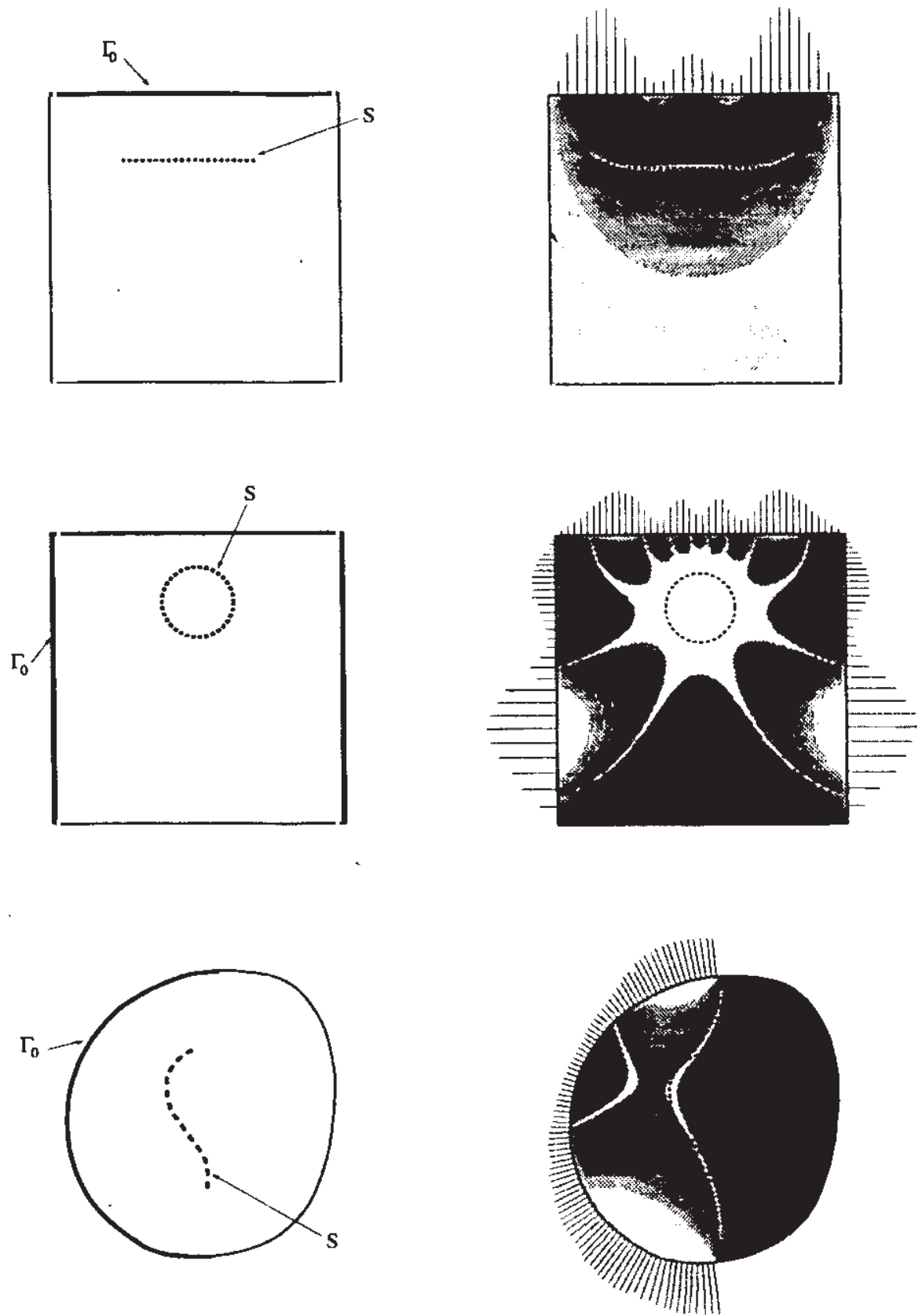

FIG. 5. Boundary control with $y_{1} h=1$. Examples 1 (top), 2 (middle) and 3 (bottom). Right: Control shown as normals. Solution in gray scale, level set $1 \pm 0.03$ white. 


\section{References}

[Ag-Do-Ni] S. Agmon, A. Douglis, and L. Nirenberg, Estimates Near The Boundary for Solutions of Elliptic Partial Differential Equations Satisfying General Boundary Conditions, I, Communications on Pure and Applied Mathematics, Vol. 12, p. 623-727, 1959.

[Br] H. Brezis, Analyse Fonctionnelle, Théorie et Applications, Masson, Paris, 1987.

[Ca-Gl-Li] C. Carthel, R. Glowinski and J.-L. Lions, On exact and approximate boundary controllabilities for the heat equation: a numerical approach, J. Optim. Theory Appl. 82, 3, p. 429-484, 1994.

[De] P. Demeestère, Méthode des sentinelles : Etude comparative et application à l'identification d'une frontière. Contrôle du temps d'explosion d'une équation de combustion. Annexe A: Régularisation de Fenchel et régularisation de Tychonov, $\mathrm{PhD}$ Thesis, Université de Technologie de Compiègne, 1997.

[Ek-Te] Y. Ekeland and R. Temam, Analyse convexe et problèmes variationnelsDunod, Gauthier-Villars, Paris, 1974.

[Fa-Le] C. Fabre and G. Lebeau, Prolongement unique des solutions de l'équation de Stokes, Comm. Partial Differential Equations 21, 3-4, p. 573-596, 1996.

[Fa-Pu-Zu1] C. Fabre, J.-P. Puel and E. Zuazua, Contrôlabilité approchée de l'équation de la chaleur semi-linéaire, C. R. Acad. Sci. Paris, t.315, série $I_{\text {, }}$ p. 807-812,1992.

[Fa-Pu-Zu2] C. Fabre, J.-P. Puel and E. Zuazua, Contrôlabilité approchée de l'équation de la chaleur linéaire avec des contrôles de norme $L^{\infty}$ minimale, C. R. Acad. Sci. Paris, t.316, série I, p. 679-684, 1993.

[Fa-Pu-Zu3] C. Fabre, J.-P. Puel and E. Zuazua, Approximate Controllability of the semilinear heat equation, Proc. of the Royal Soc. of Edinburgh, 125A, p. 31-61, 1995.

[Fe] E. Fernández-Cara, Null controllability of the semilinear heat equation, ESAIM: Control, Optimization and Calculus of Variations. Vol. 2, p. 87-103, 1997.

[Gi-Ra] V. Girault and P.-A. Raviart, Finite Element Methods for NavierStokes Equations. Theory and Algorithms, Springer Series in Computational Mathematics 5, Springer-Verlag, Berlin, 1986. 
[Gl-Li-Li] R. Glowinski, C.H. Li and J.-L. Lions, A numerical approach to the exactly boundary controlability of the wave equation. Dirichlet controls, description of numerical methods, Houston, Department of Mathematics University of Houston, 1988.

[Gl-Li-Tr] R. Glowinski, J.-L. Lions and Trémolières, Analyse Numérique des inéquations variationnelles, Paris, Dunod, 1976.

[He] J. Henry, Etude de la contrôlabilité de certaines équations paraboliques, Thèse d'Etat de l'université Paris VI, 1978.

[La-Ur] O. A. Ladyzenskaya and N. N. Uraltceva, Equations aux Derivées Partielles de Type Elliptique, Monographies Universitaires de Mathématiques, Dunod, Paris, 1968.

[Li1] J.-L. Lions, Exact controllability, stabilization and perturbations for distributed systems, SIAM, Boston, 1986, SIAM Rev., 30, p. $1-68,1988$.

[Li2] J.-L. Lions, Rernarques sur la contrôlabilité approchée, Proceedengs of "Jornadas Hispano Francesas sobre control de Sistemas Distribuidos", University of Malaga, Spain, 1990.

[Li3] J.-L. Lions, Remarks on approximate controllability, Israel J. Math. 1992.

[Li-Ma] J.-L. Lions and E. Magenes, Problèmes aux limites non homogènes et applications, Vol 1., Dunod, 1968.

[Mi] C. Miranda, Partial Differential Equations of Elliptic Type, Second revised edition, Springer-Verlag, Berlin, 1970.

[Ne] J. Nečas, Les Méthodes Directes en Théorie des Equations Elliptiques, Masson, Paris, 1967.

[Os-Pul] A. Osses and J.-P. Puel, Some extensions of approximate controllability results to inverse problems, ESAIM Proceedings: Elaticité, Viscoelasticité et Contrôle Optimal. Huitièmes Entretiens du Centre Jacques Cartier, 1, p. 157-168, 1996.

[Os-Pu2] A. Osses and J.-P. Puel, Boundary controllability of the stationary Stokes system with linear convection observed on an interior curve, To appear in J. Optim. Theory Appl.

[Pr-Te] W. Press, S. Teukolsky, W. Vetterling and B. Flannery, Numerical Recipes in $\mathrm{C}$, the art of scientific computing. Cambridge, Cambridge University Press, 1992. 
[Sa-Sc] J.-C. Saut and B. Scheurer, Sur l'unicité du problème de Cauchy et le prolongement unique des équations elliptiques à coefficients non localement bornés, Journal of Differential Equations 43, 28$43,1982$.

Centre de Mathématiques Appliquées Ecole Polytechnique,

91128 Palaiseau Cedex

Paris (FRANCE)

Université de Versailles Saint-Quentin

and

Centre de Mathématiques Appliquées

Ecole Polytechnique,

91128 Palaiseau Cedex

Paris (FRANCE)

Recibido: 11 de Febrero de 1998

Revisado: 15 de Abril de 1997 